\title{
A dark matter profile to model diverse feedback-induced core sizes of $\Lambda$ CDM haloes
}

\author{
Alexandres Lazar ${ }^{\circledR},{ }^{1 \star}$ James S. Bullock ${ }^{\circledR},{ }^{1}$ Michael Boylan-Kolchin ${ }^{\circledR}, 2$ T. K. Chan ${ }^{\circledR}$, 3,4 \\ Philip F. Hopkins ${ }^{\circledR}, 5$ Andrew S. Graus, ${ }^{2}$ Andrew Wetzel ${ }^{\circledR}, 6$ Kareem El-Badry ${ }^{\circledR},{ }^{7}$ Coral Wheeler, ${ }^{4}$ \\ Maria C. Straight, ${ }^{8,2}$ Dušan Kereš, ${ }^{4}$ Claude-André Faucher-Giguère ${ }^{\circledR},{ }^{9}$ Alex Fitts ${ }^{\circledR 2}$ \\ and Shea Garrison-Kimmel ${ }^{\circledR 5}$ \\ ${ }^{1}$ Department of Physics and Astronomy, University of California, Irvine, CA 92697 USA \\ ${ }^{2}$ Department of Astronomy, The University of Texas at Austin, 2515 Speedway, Stop C1400, Austin, TX 78712-1205, USA \\ ${ }^{3}$ Institute for Computational Cosmology, Durham University, South Road, Durham DH1 3LE, UK \\ ${ }^{4}$ Department of Physics, Center for Astrophysics and Space Science, University of California at San Diego, 9500 Gilman Dr., La Jolla, CA 92093, USA \\ ${ }^{5}$ California Institute of Technology, TAPIR, Mailcode 350-17, Pasadena, CA 91125, USA \\ ${ }^{6}$ Department of Physics, University of California, Davis, CA 95616, USA \\ ${ }^{7}$ Department of Astronomy and Theoretical Astrophysics Center, University of California Berkeley, Berkeley, CA 94720, USA \\ ${ }^{8}$ Department of Physics, Whitworth University, 300 West Hawthorne Road, Spokane, Washington 99251, USA \\ ${ }^{9}$ Department of Physics and Astronomy and CIERA, Northwestern University, 2145 Sheridan Road, Evanston, IL 60208, USA
}

Accepted 2020 July 7. Received 2020 June 16; in original form 2020 April 22

\begin{abstract}
We analyse the cold dark matter density profiles of 54 galaxy haloes simulated with Feedback In Realistic Environments (FIRE)-2 galaxy formation physics, each resolved within 0.5 per cent of the halo virial radius. These haloes contain galaxies with masses that range from ultrafaint dwarfs $\left(M_{\star} \simeq 10^{4.5} \mathrm{M}_{\odot}\right)$ to the largest spirals $\left(M_{\star} \simeq 10^{11} \mathrm{M}_{\odot}\right)$ and have density profiles that are both cored and cuspy. We characterize our results using a new, analytic density profile that extends the standard two-parameter Einasto form to allow for a pronounced constant density core in the resolved innermost radius. With one additional core-radius parameter, $r_{\mathrm{c}}$, this three-parameter core-Einasto profile is able to characterize our feedback-impacted dark matter haloes more accurately than other three-parameter profiles proposed in the literature. To enable comparisons with observations, we provide fitting functions for $r_{\mathrm{c}}$ and other profile parameters as a function of both $M_{\star}$ and $M_{\star} / M_{\text {halo. }}$. In agreement with past studies, we find that dark matter core formation is most efficient at the characteristic stellar-to-halo mass ratio $M_{\star} / M_{\text {halo }} \simeq 5 \times 10^{-3}$, or $M_{\star} \sim 10^{9} \mathrm{M}_{\odot}$, with cores that are roughly the size of the galaxy half-light radius, $r_{\mathrm{c}} \simeq 1-5 \mathrm{kpc}$. Furthermore, we find no evidence for core formation at radii $\gtrsim 100 \mathrm{pc}$ in galaxies with $M_{\star} / M_{\text {halo }}<5 \times 10^{-4}$ or $M_{\star} \lesssim 10^{6} \mathrm{M}_{\odot}$. For Milky Way-size galaxies, baryonic contraction often makes haloes significantly more concentrated and dense at the stellar half-light radius than DMO runs. However, even at the Milky Way scale, FIRE-2 galaxy formation still produces small dark matter cores of $\simeq$ $0.5-2 \mathrm{kpc}$ in size. Recent evidence for a $\sim 2 \mathrm{kpc}$ core in the Milky Way's dark matter halo is consistent with this expectation.
\end{abstract}

Key words: galaxies: evolution-galaxies: formation-dark matter.

\section{INTRODUCTION}

The theory of Cold Dark Matter with the inclusion of the cosmological constant $(\Lambda \mathrm{CDM})$ has been the benchmark paradigm in cosmological studies, as its framework has been successful in modelling the distribution of large-scale structure of our Universe. However, on small scales, there are potential inconsistencies between predictions made by the $\Lambda \mathrm{CDM}$ paradigm and what is observed in real galaxies. One of these inconsistencies concerns the distribution of dark matter in centres of galaxies. This known as the cusp-core problem: dark matter haloes simulated without baryons in $\Lambda$ CDM have cusped dark matter densities at small radii, i.e. $\rho(r) \propto r^{\alpha}$

^E-mail: aalazar@uci.edu with $\alpha \sim-1$ (Dubinski \& Carlberg 1991; Navarro, Frenk \& White 1997; Navarro et al. 2004), while observations of some dark matter dominated galaxies appear to suggest profiles are better described by constant density cores at small radii, i.e. $\alpha \sim 0$ (Flores $\&$ Primack 1994; Moore 1994; Salucci \& Burkert 2000; Swaters et al. 2003; Gentile et al. 2004; Spekkens, Giovanelli \& Haynes 2005; Walter et al. 2008; Oh et al. 2011; Relatores et al. 2019). Another potentially related discrepancy is called the Too Big to Fail problem (BoylanKolchin, Bullock \& Kaplinghat 2011): Milky Way satellite galaxies are observed to have much smaller inner dark matter densities compared to the surplus of subhaloes predicted from [dark matter only (DMO)] cosmological $N$-body simulations. This problem also persists in other dwarf galaxies of the Local Group and local field (Garrison-Kimmel et al. 2014; Tollerud, Boylan-Kolchin \& Bullock 2014; Papastergis et al. 2015). 
Most of the above-mentioned problems were posed from DMO simulations, which lack the effects of baryons. One way galaxy formation can affect dark matter is by boosting central dark matter densities as a result of baryons clustering at the centre of the halo (Blumenthal et al. 1986). This denoted as 'baryonic contraction' in the literature and it is an effect that is particularly important for Milky Way-mass galaxies (e.g. Gnedin et al. 2004; Chan et al. 2015). Alternatively, the inner dark matter density can decrease in response to repetitive energetic outflows from stellar feedback, a process often referred to as 'feedback-induced core formation', and one that is most effective in galaxies that are somewhat smaller than the Milky Way (Navarro, Eke \& Frenk 1996; Read \& Gilmore 2005; Governato et al. 2010, 2012; Pontzen \& Governato 2012; Teyssier et al. 2013; Di Cintio et al. 2014a; Brook \& Di Cintio 2015; Chan et al. 2015; Tollet et al. 2016). Another possibility is that dynamical friction from small accretion events (El-Zant, Shlosman \& Hoffman 2001; Tonini, Lapi \& Salucci 2006; Romano-Díaz et al. 2008; Goerdt et al. 2010; Cole, Dehnen \& Wilkinson 2011) can flatten the dark matter density profile.

The effects of feedback on core formation depend sensitively on the total amount and precise nature of star formation. For example, Peñarrubia et al. (2012) showed that galaxies with too few stars (and therefore, too few supernovae) are unlikely to have feedback-induced cores owing to an insufficient amount energy from supernovae to substantially transform the dark matter profile. Mashchenko, Couchman \& Wadsley (2006) showed that concentrated star formation episodes that are spatially displaced from halo centres can drive bulk gas flows, alter dark matter particle orbits, and increase the likelihood for dark matter core formation. Time-repetitive 'bursty' star formation also affects core formation, allowing for dark matter particle orbits to be affected significantly over time as gas is expelled and re-accreted in the baryon cycle (Pontzen \& Governato 2012). The timing of star formation relative to dark matter halo growth can also affect core formation; in cases where dark matter rich mergers occur after core-producing star formation, cusps can be reborn (Oñorbe et al. 2015). Dark matter core formation is seen in many fully self-consistent cosmological simulations that resolve star formation on small spatial scales (e.g. Governato et al. 2010; Munshi et al. 2013; Brooks \& Zolotov 2014; Madau, Shen \& Governato 2014; Oñorbe et al. 2015; El-Badry et al. 2016; Tollet et al. 2016; Fitts et al. 2017). One common aspect of these simulations is that they have relatively high gas density thresholds for star formation. Cosmological simulations with lower density thresholds for star formation, e.g. APOSTLE and Auriga (Bose et al. 2019), have been shown to not produce dark matter cores. The dependence of feedbackinduced core formation on the star formation density threshold has been studied in more detail by Dutton et al. (2019) and BenítezLlambay et al. (2019). Both concluded that density thresholds higher than the mean ISM density, which allows for some ISM phase structure and clustered star formation as observed, is necessary in forming feedback-induced cores.

Di Cintio et al. (2014a) studied the relationship between the inner local density slope of dark matter, $\alpha$, and the stellar mass fraction, $M_{\star} / M_{\text {halo }}$, of simulated galaxies from the MUGS (Stinson et al. 2010) and MaGICC (Brook et al. 2012; Stinson et al. 2012) simulations for a wide range stellar mass systems, $M_{\star} \simeq 10^{5-11} \mathrm{M}_{\odot}$. They found that core formation is a strong function the mass-ratio of stars formed to total halo mass and demonstrated that there is a characteristic mass-ratio for efficient core formation $M_{\star} / M_{\text {halo }} \simeq$ $5 \times 10^{-3}$, above and below which galaxy haloes approach the cuspy behaviour associated with DMO simulations. Chan et al. (2015) used galaxies of stellar masses, $M_{\star}=10^{3-11} \mathrm{M}_{\odot}$, from the Feedback
In Realistic Environments (FIRE)-1 suite (Hopkins et al. 2014) to study feedback-induced core formation and found similar results. Tollet et al. (2016) used the Numerical Investigation of Hundred Astrophysical Objects (NIHAO) suite (Wang et al. 2015) for a wide range of halo masses, $M_{\text {halo }}=10^{10-12} \mathrm{M}_{\odot}$ and further confirmed this qualitative phenomena. Recently, Macciò et al. (2020) extended the work of Tollet et al. (2016) with the inclusion of black hole feedback for galaxies spanning eight orders in magnitude in stellar mass.

The above-mentioned simulation groups agree on a few additional qualitative points. First, feedback typically does not produce significant deviations from cuspy DMO predictions in the smallest galaxies: $M_{\star} / M_{\text {halo }}<10^{-4}\left(M_{\star} \lesssim 10^{6} \mathrm{M}_{\odot}\right.$, typically), as expected on energetic grounds (Peñarrubia et al. 2012; Garrison-Kimmel et al. 2013). Secondly, dark matter haloes become more cored as $M_{\star} / M_{\text {halo }}$ increases up until $M_{\star} / M_{\text {halo }} \simeq 5 \times 10^{-3}$, which is the region of peak core formation. These haloes are not well modelled by cuspy density profiles and must be described by an alternative dark matter profile that has a pronounced flattening in slope at small radii. In higher mass haloes, $M_{\text {halo }} \simeq 10^{12} \mathrm{M}_{\odot}$, baryonic contraction actually makes haloes denser at the stellar half-mass radius than DMO simulations would suggest. However, Chan et al. (2015) found that within this radius, small cores are often present even within baryonically contracted $10^{12} \mathrm{M}_{\odot}$ haloes.

The analysis done in Di Cintio et al. (2014b) explored a general five-parameter density profile to characterize haloes with either cuspy or cored inner density profiles. In addition to a characteristic radius and density, this profile had three shape parameters: $\alpha, \beta$, and $\gamma$ (Zhao 1996). They found that the values of the three shape parameters varied regularly as a function of the $M_{\star} / M_{\text {halo }}$ and provided fitting functions that captured these trends. Therefore, given $M_{\star} / M_{\text {halo }}$, the Di Cintio et al. (2014b) profile reduces to a two free-parameter function that may be used to compare predictions with observations in a fairly straightforward manner.

The $\alpha \beta \gamma$-profile can be regarded as a generalization of the Navarro et al. (1997; NFW) profile, which provides a good fit to DMO simulations. Since DMO simulations have traditionally been characterized by the NFW profile, there have been attempts to modify the NFW form by allowing for a constant density coreradius parameter $r_{\mathrm{c}} \equiv r_{\text {core }}$. For example, Peñarrubia et al. (2012) suggested a three-parameter core profile: the classic NFW profile with a core radius in the inner radial regions of the halo. Read, Agertz \& Collins (2016) derive a core profile starting with an NFW form by connecting core formation to features of star formation efficiency and the stellar half-mass radius. More recently, Freundlich et al. (2020) used NIHAO to explore a constrained version of the $\alpha \beta \gamma$ profile that has three-parameters, the 'Dekel + ' profile (Dekel et al. 2017), with a variable inner slope and concentration parameter.

In what follows, we revisit the question of dark matter halo density profiles in cosmological galaxy formation simulations using the FIRE-2 feedback model (Hopkins et al. 2018). The simulations we consider herein allow us to resolve to within 0.5 per cent of the halo virial radius in haloes that produce galaxies spanning six orders of magnitude in stellar mass. We introduce a new analytic density profile, the 'core-Einasto', that extends the Einasto (1965) form by adding one free parameter, a physical core radius, $r_{\mathrm{c}}$. It is well known that the two-parameter Einasto profile provides a better fit to DMO simulations than the two-parameter NFW (Navarro et al. 2004; Wang et al. 2019). Similarly, we find that the three-parameter core-Einasto profile provides a better fit to FIRE-2 haloes than two popular threeparameter versions of generalized double power law profiles: the 
Dekel + profile (Dekel et al. 2017; Freundlich et al. 2020) and a cored extension of the NFW (Peñarrubia et al. 2012). We also find that the two-parameter Di Cintio et al. (2014b) profile is not a good fit to our feedback-affected haloes.

In addition to providing a better fit to our FIRE-2 haloes than other three-parameter profiles, the core-Einasto profile utilizes a physically meaningful core-radius parameter, $r_{\mathrm{c}}$. The numerical value of $r_{\mathrm{c}}$ matches well to the radius where a visual profile begins to flatten towards a constant density. The combination of accuracy, intuitive parameters, and ease-of-use will hopefully allow our threeparameter core-Einasto profile to become a useful tool for comparing predictions to observations.

This article is structured as follows: Section 2 discusses our sample of high-resolution galaxies simulated with FIRE-2 physics along with their relevant properties. We also discuss the numerical intricacies considered for our galaxies. Section 3 revisits the analysis of correlations between $\alpha$ and $M_{\star} / M_{\text {halo }}$ for our sample of galaxies and dark matter haloes. In Section 4 , we introduce the cored version of the classic Einasto profile used to model $\Lambda \mathrm{CDM}$ haloes. We use the properties of these profiles to provide constraints on dark matter cores as a function and of $M_{\star} / M_{\text {halo }}$. We summarize our results and discuss potential uses for observational and cosmological studies in Section 5. The appendix includes five sections: Appendix A has expressions for fitting parameters as a function of stellar mass; Appendix B derives analytical expressions for the mass and gravitational potential implied by the core-Einasto profile; Appendix $\mathrm{C}$ has a four-parameter core-Einasto extension that better accounts for adiabatic contraction in Milky Way size haloes; Appendix D presents comparisons to fits with alternative three-parameter profiles and also presents fits for the five-parameter $\alpha \beta \gamma$ form; and Appendix E provides tables that list all halo properties and best-fitting profile parameters for each halo in our sample.

\section{NUMERICAL METHODOLOGY}

In this section, we briefly describe the suite of high-resolution simulations used in our analysis. We discuss the FIRE-2 model for full galaxy formation physics in Section 2.1, the numerical parameters used in our high-resolution simulations in Sections 2.2 and 2.5, and present the halo sample used in this analysis in Section 2.3. The numerical simulations presented here are all part of the FIRE project ${ }^{1}$ and are listed in Table E1 at the end of this article.

\subsection{The FIRE-2 model}

Our simulations were run using the multimethod code GIZMO (Hopkins 2015), with the second-order mesh-free Lagrangian-Godunov finite mass (MFM) method for hydrodynamics. GIZMO utilizes an updated version of the $\mathrm{pm}+$ tree algorithm from GADGET-3 (Springel 2005) to calculate gravity and adopts fully conservative adaptive gravitational softening for gas (Price \& Monaghan 2007). The FIRE2 model (Hopkins et al. 2018), which is an updated version of the FIRE-1 feedback scheme from Hopkins et al. (2014), is used to implement star formation and stellar feedback physics. Gas and gravitational physics implemented are discussed in complete detail in Hopkins et al. (2018). Here, we discuss in brief detail the feedback physics relevant to core formation.

The simulations presented here tabulate the relevant ionization states and cooling rates from a compilation of CLOUDY runs (Ferland

\footnotetext{
${ }^{1}$ The FIRE project website: http://fire.northwestern.edu.
}

et al. 1998), accounting for gas self-shielding. The gas cooling mechanisms follow the cooling rates of $T=10-10^{10} \mathrm{~K}$; these include metallicity-dependent fine-structure atomic cooling, lowtemperature molecular cooling, and high-temperature metal-line cooling that followed 11 separately tracked species. Gas is heated and ionized throughout cosmic time using the redshift-dependent UV background model from Faucher-Giguère et al. (2009) that ionizes and heats gas in an optically thin approximation and uses an approximate prescription to account for self-shielding of dense gas using a Sobolev/Jeans-length approximation. Stars are formed in Jeans-unstable, molecular gas regions at densities $n_{\mathrm{H}} \geq 10^{3} \mathrm{~cm}^{-3}$, with 100 per cent instantaneous efficiency per local free-fall time in dense gas. Each star particle is an assumed stellar population with a Kroupa (2001) IMF that inherits its metallicity from its parent gas particle and has an age determined by its formation time. The stellar feedback implemented includes stellar winds, radiation pressure from young stars, Type II and Type Ia supernovae, photoelectric heating, and photoheating from ionizing radiation. Feedback event rates, luminosities, energies, mass-loss rates, and other quantities are tabulated directly from stellar evolution models (STARBURST99; Leitherer et al. 1999).

\subsection{Numerical simulations}

All simulations in this analysis use a zoom-in technique (Oñorbe et al. 2014) to reach high resolutions in a cosmological environment by constructing a convex-hull region and refining it in progressively higher resolution shells until the desired resolution is reached in the innermost region. All initial conditions are generated with MUSIC (Hahn \& Abel 2011) and then the simulations are evolved from redshifts $z \approx 100$ to $z=0$ assuming a flat $\Lambda$ CDM cosmology. We note that the cosmological parameters in each of the simulations vary to some degree, but remain consistent with Planck Collaboration XIII (2016). Across our entire simulation sample: $h=0.68-0.71$, $\Omega_{\Lambda}=1-\Omega_{m}=0.69-0.73, \Omega_{b}=0.0455-0.048, \sigma_{8}=0.801-0.82$, $n_{s}=0.961-0.97$. In post-processing, haloes are identified using the phase-space halo finder ROCKSTAR (Behroozi, Wechsler \& Wu 2013), which uses adaptive, hierarchical refinement of the friends-of-friends groups in 6D phase-space and one time dimension. This results in robust tracking of haloes and subhaloes (Srisawat et al. 2013).

\subsection{Halo sample and nomenclature}

Throughout this paper, dark matter haloes are defined as spherical systems with virial radius, $r_{\text {vir }}$, inside which the average density is equal to $\Delta_{\text {vir }}(z) \rho_{\text {crit }}(z)$. Here, $\rho_{\text {crit }}(z):=3 H^{2}(z) / 8 \pi G$ is the critical density of the universe and $\Delta_{\text {vir }}(z)$ is the redshift evolving virial overdensity defined in Bryan \& Norman (1998). The virial mass of a dark matter halo, denoted by $M_{\text {halo }}$, is then defined as the dark matter mass within $r_{\text {vir }}$. The stellar mass of the galaxy, $M_{\star}$, is then taken to be the total sum of the stellar particles inside 10 per cent of $r_{\text {vir }}$. It follows that the 3D stellar half-mass radius, $r_{1 / 2}$, is the radius that encloses half of the defined stellar mass. Finally, we refer to the 'stellar fraction' of the halo as the ratio between the quantified stellar mass and the halo mass: $M_{\star} / M_{\text {halo }}$.

Fig. 1 outlines our sample of galaxies, where just the dark matter halo masses (from the FIRE-2 runs) are plotted against $M_{\star}$. We compare our sample with the the abundance-matching relations presented in (zero scatter; Garrison-Kimmel et al. 2017) and Behroozi et al. (2019) as the blue and pink curves, respectively, showing the best-fitting median abundance-matching relations. Table E1 lists all of the haloes galaxies in this paper, including their $z=0$ 


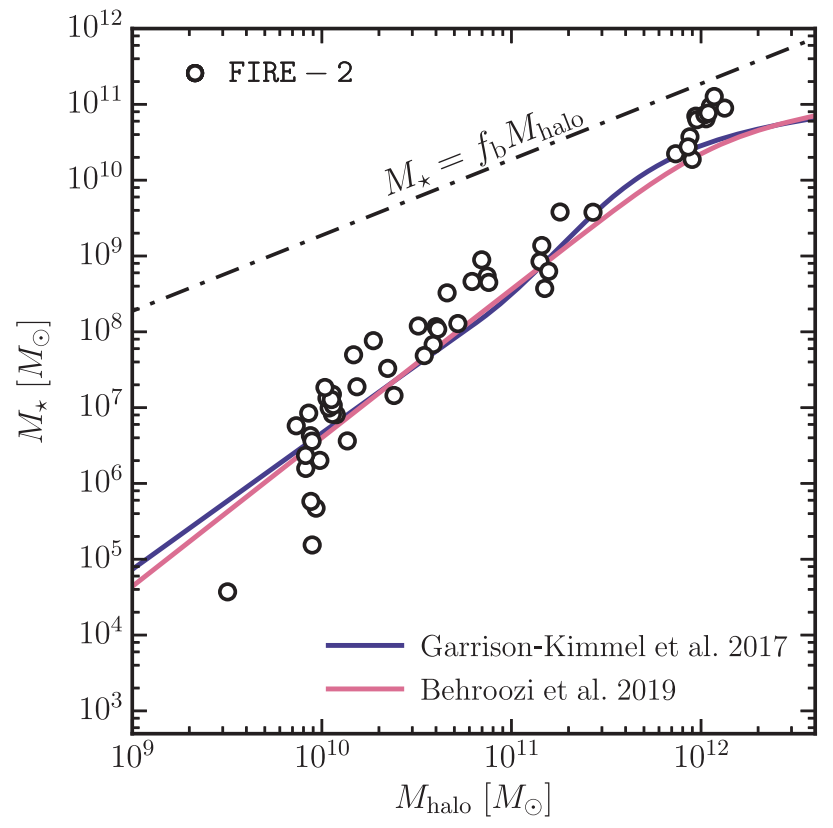

Figure 1. Stellar-to-halo mass relations. The white points show galaxies from the FIRE-2 simulations studied in this paper. The curves are the median abundance matching relations presented in (zero scatter; Garrison-Kimmel et al. 2017; blue) and Behroozi et al. (2019; pink).

properties from the FIRE-2 runs. Given our large sample, we chose to divide our galaxy sample into four convenient classifications of objects using the convention from Bullock \& Boylan-Kolchin (2017): ${ }^{2}$

(i) Ultrafaint dwarfs: Defined to have stellar masses of $M_{\star} \approx$ $10^{2-5} \mathrm{M}_{\odot}$ at $z=0$. These are analogues of galaxies to be detected within limited local volumes around M31 and the Milky Way.

(ii) Classical dwarfs: Defined to have stellar masses of $M_{\star} \approx$ $10^{5-7} \mathrm{M}_{\odot}$ at $z=0$. These are analogues of the faintest galaxies known prior to SDSS.

(iii) Bright dwarfs: Defined to have stellar masses of $M_{\star} \approx$ $10^{7-10} \mathrm{M}_{\odot}$ at $z=0$. These are analogues of the faintest galaxies that can be seen in wide-field galaxy surveys.

(iv) Milky Way-mass haloes: Defined to host spiral galaxies with stellar mass of $M_{\star} \approx 10^{10-11} \mathrm{M}_{\odot}$ at $z=0$. At the peak of abundancematching relation, this maps to the generally accepted range in Milky Way-mass haloes of $M_{\text {halo }}=[0.8-2.4] \times 10^{12}$. Hereafter, we abbreviate Milky Way as 'MW'.

Lastly, each zoomed-in halo run with full FIRE-2 physics has an analogous DMO version. The individual dark matter particle masses in the DMO versions are larger by a factor of $\left(1-f_{\mathrm{b}}\right)^{-1}$ in these runs, where $f_{\mathrm{b}}:=\Omega_{\mathrm{b}} / \Omega_{m}$ is the cosmic baryon fraction, but the initial conditions are otherwise identical. The density profiles quoted from the DMO simulations have been scaled $m_{\mathrm{p}} \rightarrow\left(1-f_{\mathrm{b}}\right) m_{\mathrm{p}}$ to roughly account for the exclusion of the baryons. Other quantities are also adjusted accordingly: $\rho(r) \rightarrow\left(1-f_{\mathrm{b}}\right) \rho(r), M_{\text {halo }}(<r) \rightarrow$ $\left(1-f_{\mathrm{b}}\right) M_{\text {halo }}(<r)$ and $V_{\text {circ }}(r) \rightarrow \sqrt{1-f_{\mathrm{b}}} V_{\text {circ }}(r)$, for all of the results analysed in the DMO runs. This provides a simple comparison set to understand the additional effects of energetic feedback seen in our FIRE-2 runs.

\footnotetext{
${ }^{2}$ Note that these classifications are based on galaxies that span specific stellar
} mass ranges.

\subsection{Radial profiles}

For each main halo identified by ROCKSTAR, the centre of the halo is quantified through a 'shrinking spheres' iteration scheme (Power et al. 2003; Navarro et al. 2004): The centre of mass of particles is computed in a sphere and then has its radius reduced by half and re-centered on the new centre of mass. This is done successively until the sphere contains one thousand particles. The final centre of mass position is determined at this last iteration. For our galaxies, this is done for the combined star and dark matter particles found inside the virial radius, while the centre of mass for the DMO analogues are done with only dark matter inside the halo. ${ }^{3}$ The spherically averaged local density profile, $\rho(r)$, is constructed in 35 logarithmically spaced bins over $r \in$ [0.005-1] $\times r_{\text {vir }}$. We expected systematic uncertainties in the binned density estimates to be extremely minimal due to large number of particles in each simulation sample. Throughout the entirety of this paper, we refer to these local density profiles as the density profiles for the dark matter halo.

\subsection{Region of numerical convergence}

We expect the innermost regions of our simulated haloes to be affected by numerical relaxation. With a variety of galaxies simulated at different resolutions, we must account for resolution differently in each simulation. We do so using the method specified in Power et al. (2003), where the effective resolution of cosmological simulations is related to the radius where the two-body relaxation timescale, $t_{\text {relax }}$, becomes shorter than the age of the universe, $t_{0}$. Precisely, the radius at which numerical convergence is achieved, $r_{\text {conv }}$, is dependent on the number of enclosed particles, $N(<r)$, as well as the mean density enclosed at the associated radius, $\bar{\rho}(r)=3 M(<r) / 4 \pi r^{3}$, where $M(<r)$ is the total mass contained within radius $r$. Therefore, $r_{\text {conv }}$ is governed by the following equation:

$\frac{t_{\text {relax }}(r)}{t_{0}}=\frac{\sqrt{200}}{8} \frac{N}{\ln N}\left[\frac{\bar{\rho}(r)}{\rho_{\text {crit }}}\right]^{-1 / 2}$.

A rigorous study of the numerical convergence for DMO haloes and the FIRE-2 galaxies (dark matter with baryons) has been discussed in detail in Hopkins et al. (2018). There, the convergence has been gauged as a function of mass resolution, force resolution, time resolution, and so on.

For the DMO simulations, convergence was shown to be well resolved to the radius at which the criterion satisfies $t_{\text {relax }}>0.6 t_{0}$ with $<1$ per cent resolution level deviations. This typically equates to $\sim 2000$ particles and is more conservative for the ranges of resolution levels analysed in our halo sample. However, even at $\sim 200$ particles (resulting in a factor $\sim 2$ smaller radius of convergence), the convergence is good to $\sim 10$ per cent in the density profile. Hereafter, we adopt $t_{\text {relax }}=0.6 t_{0}$ as our resolution criterion to maintain consistency across all of our simulations. We define $r_{\text {conv }}:=r_{0.6}^{\mathrm{DM}}$ to be the radius at which the resolution criterion is fulfilled for the $D M O$ analogues of each sample halo, meaning that $r>r_{\text {conv }}$ is our best estimate of the numerically converged region. In Hopkins et al. (2018), convergence for simulations ran with baryons can be much better or worse in comparison to their DMO analogues, but convergence is entirely dominated by the convergence from the baryons. So, in the context of our galaxies, the criterion of convergence has much more to do with the star formation dynamics

\footnotetext{
${ }^{3}$ We also compared our results with centres defined as the most bound dark matter particle in the halo determined by ROCKSTAR. We find no qualitative differences in our final results.
} 


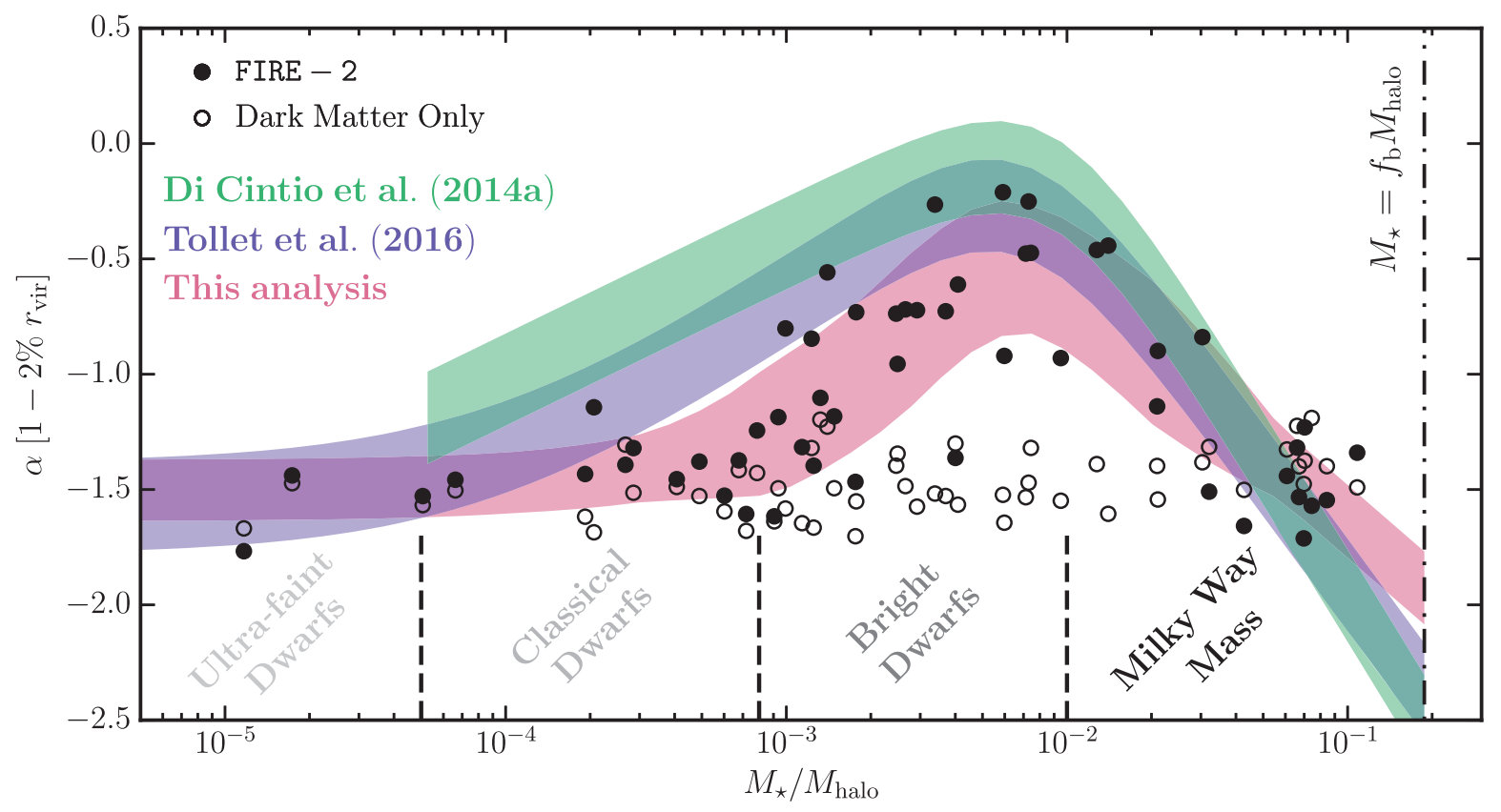

Figure 2. The impact of feedback physics on the inner dark matter densities. Shown is the inner dark matter density slope, $\alpha$, averaged over [0.01-0.02] $\times r_{\mathrm{vir}}$, as a function of the stellar mass fraction, $M_{\star} / M_{\text {halo }}$, at $z=0$. Cored profiles have $\alpha \sim 0$, while cuspy inner density profiles have lower values of $\alpha \lesssim-1$. The open circles are the DMO analogues, which all have $\alpha \approx-1.5$ as expected from a NFW profile. The pink-shaded region shows the $1 \sigma$ dispersion about the smoothed binned median. As a comparison, the fits from (green; Di Cintio et al. 2014a) and (blue; Tollet et al. 2016) are also plotted using a constant width of $\Delta \alpha= \pm 0.2$ relative to the mean relation (Tollet et al. 2016). The curve from Di Cintio et al. (2014a) was only fitted to down to a stellar mass fraction of $M_{\star} / M_{\text {halo }} \simeq 4 \times 10^{-5}$, so we restrict the curve to that mass limit. The dispersion in $\alpha$ increases from the stellar mass fraction from $M_{\star} / M_{\text {halo }} \gtrsim 10^{-4}$, the regime of classical dwarfs and the brightest dwarfs, to the MW-mass haloes with $M_{\star} / M_{\text {halo }} \simeq 10^{-1}$. Feedback-induced core formation peaks at $M_{\star} / M_{\text {halo }} \simeq 5 \times 10^{-3}$, the regime of the brightest dwarfs. At $M_{\star} / M_{\text {halo }} \lesssim 10^{-4}$, the regime of classical dwarfs and ultrafaints, the impact of stellar feedback is negligible.

and converging baryonic physics rather than having to do with the number of particles enclosing a specific region. With this, $r_{\text {conv }}$ from the DMO analogues are applied to the galaxies of the FIRE-2 haloes throughout this paper as a conservative estimate. For more details regarding the numerical convergence study of FIRE-2 haloes, we refer to Hopkins et al. (2018).

\section{STELLAR FRACTION RELATION WITH THE INNER DENSITY SLOPE}

We begin by comparing our catalog of galaxies with previous results in the literature. The stellar mass fraction, which we define as the ratio between the stellar mass and halo mass, $M_{\star} / M_{\text {halo }}$, has a relationship with the slope of the dark matter density profile found at the innermost radii (Di Cintio et al. 2014a; Chan et al. 2015; Tollet et al. 2016). Following the convention of Di Cintio et al. (2014a), the effect of feedback on the inner dark matter halo density can be captured by exploring the best-fitting power law for the dark matter density profile over a specific radial range, $\rho(r) \propto r^{\alpha}$. Di Cintio et al. (2014a) suggested using $\alpha$ fitted over the radial range $r \in[0.01-0.02] \times r_{\text {vir }}$ since the lower limit of 1 per cent of $r_{\text {vir }}$ satisfied the Power et al. (2003) radius criterion of convergence for the majority of their halo sample.

Fig. 2 summarizes the relation between $\alpha$ and the stellar mass fraction at $z=0$ for our simulations and compares to results from (Di Cintio et al. 2014a; the green band) and (Tollet et al. 2016; the blue band). The analysis performed in green (Di Cintio et al. 2014a) included only stellar mass fractions down to $M_{\star} / M_{\text {halo }} \simeq$ $4 \times 10^{-5}$, so we restrict their curve to that limit. The differences between the two curves included differences in cosmological models used, as noted in (Tollet et al. 2016). The black-filled circles are our simulated FIRE-2 galaxies and the black open circles are the results for the DMO simulations (for which we use the stellar mass of their galaxy analogues). For all values of $M_{\star} / M_{\text {halo }}$, the DMO analogues are cuspy, with $\alpha \approx-1.5$, which is expected when assuming the behaviour of an analytic NFW profile along with scatter induced by the mass-concentration relation (see Bullock \& Boylan-Kolchin 2017).

The pink band captures our results using the fitting-formula shape suggested by Tollet et al. (2016):

$\alpha(x)=n-\log _{10}\left[n_{1}\left(1+\frac{x}{x_{1}}\right)^{-\beta}+\left(\frac{x}{x_{0}}\right)^{\gamma}\right]$,

where $x=M_{\star} / M_{\text {halo }}$. We find that $n=-1.60, n_{1}=0.80, x_{0}=$ $9.18 \times 10^{-2}, x_{1}=6.54 \times 10^{-3}, \beta=5$, and $\gamma=1.05$ matches our results in the median. The general purpose of this fit is to guide the eye. We also binned by $M_{\star} / M_{\text {halo }}$ to compute a rough estimate of the standard deviation found at each stellar fraction. The width of the pink band roughly corresponds to the $1 \sigma$ dispersion about the median. The width of the green and blue bands are set at a constant $\Delta \alpha= \pm 0.2$.

Ultrafaint and classical dwarf galaxies, with low stellar mass fractions of $M_{\star} / M_{\text {halo }} \lesssim 10^{-3}$, have inner densities slopes of $\alpha \approx$ -1.5 , the same as their DMO analogues. From there and increasing to $M_{\star} / M_{\text {halo }} \simeq 5 \times 10^{-3}$, the inner dark matter densities of the bright dwarf galaxies transition to more cored profiles. At $M_{\star} / M_{\text {halo }}$ $\simeq 5 \times 10^{-3}$, our galaxies reach efficient core formation (shown more directly next), with $\alpha \approx-0.25$. The diversity in core strength, as quantified by $\alpha$, is largest from $M_{\star} / M_{\text {halo }} \approx 10^{-3}$ to $5 \times 10^{-3}$, with a variance of $\Delta \alpha \approx \pm 0.35$ about the median. Note that one bright 
dwarf (m11q) at $M_{\star} / M_{\text {halo }} \simeq 4 \times 10^{-3}$ has what appears to be a cuspy central density. We checked the assembly history of this galaxy and verified that it is not particularly unusual, with its last major merger at $z \sim 2$. This galaxy does in fact have a constant density core (see Table E2 in the appendix), but at a radius $\sim 850 \mathrm{pc}$, which is smaller than 1 per cent of $r_{\text {vir }}(\sim 1500 \mathrm{pc})$, meaning that it is not detected using this $\alpha$ slope measurement. From the region of efficient core formation to MW masses, $\alpha$ decreases. The scatter in $\alpha$ remains large ( $\Delta \alpha \approx \pm 0.3$ ) until $M_{\star} / M_{\text {halo }} \approx 6 \times 10^{-2}$, which is in the range of the majority of the MW-mass haloes. The scatter is minimized at $\Delta \alpha$ $\approx \pm 0.15$ for these galaxy masses.

Our findings agree with previous results in the literature for the region of efficiently peaked core formation: $M_{\star} / M_{\text {halo }} \simeq 5 \times 10^{-3}$ (Di Cintio et al. 2014a; Chan et al. 2015; Tollet et al. 2016). While we do not have a significant sample of ultrafaint dwarfs, we find negligible core formation for $M_{\star} / M_{\text {halo }} \lesssim 10^{-4}$. The most significant difference we see with past results are (i) core formation that is less pronounced than previously reported for $M_{\star} / M_{\text {halo }} \simeq 10^{-3}\left(M_{\star} \simeq 10^{7} M_{\odot}\right)$ and (ii) more scatter in $\alpha$ within the regime of the brightest dwarfs, with $\alpha$ ranging from quite cuspy ( $\alpha \approx-1.5)$ to very cored $(\alpha \approx-0.25)$ over the small range $M_{\star} / M_{\text {halo }} \simeq[2-5] \times 10^{-3}$.

While results on $\alpha$ at $r \simeq 0.015 r_{\text {vir }}$ have proven useful for characterizing the effectiveness of core formation as a function of stellar mass fraction in dark matter haloes in the past, more recent simulations have allowed predictions at even smaller radii. This can potentially lead to small cores being unaccounted for (see Chan et al. 2015; Wheeler et al. 2019). For example, while Fig. 2 gives the impression that MW-mass haloes will have density structure similar to the DMO (NFW-like) expectation, this is only because the logslope at [0.01-0.02] $\times r_{\text {vir }}$ does not provide a complete picture. That is, while the log-slope at this radius is similar to that expected in the absence of galaxy formation, the overall density amplitude at $\sim 1$ per cent of the virial radius is higher. In fact, as we will see in the upcoming section, at even smaller radii, our MW-mass haloes have cored density profiles. ${ }^{4}$ This motivates a more complete examination into the shapes of profiles of simulated galaxy haloes.

\section{A DENSITY PROFILE FOR FEEDBACK-AFFECTED HALOES}

In this section, we present a new dark matter density profile that allows for constant density cores of the type seen in our simulated galaxy haloes. The new profile generalizes the Einasto (1965) profile, which has proven to be an excellent fit for haloes formed in DMO simulations. Our 'core-Einasto' (cEinasto) profile extends its behaviour with one free parameter - a core radius, $r_{\mathrm{c}}$. After demonstrating that this profile does sufficiently well of capturing the density structure for a majority of the FIRE-2 haloes, we follow the methodology employed in Di Cintio et al. (2014b), and provide fits for halo fitting parameters as functions of $M_{\star} / M_{\text {halo }}$ at $z=0$. In Appendix A, we provide profile parametrization as a function of galaxy stellar mass, $M_{\star}$. We note that in the course of this analysis, we explored several different options for analytic cored profiles and found that the core-Einasto form was the best of these fits. In Appendix D, we show an example comparison between the coreEinasto profile and the Peñarrubia et al. (2012; core-NFW) profile and demonstrate that core-Einasto provides a superior fit with the same number of free parameters.

${ }^{4}$ Also seen from the implementation of FIRE-1 physics for MW-mass haloes in Chan et al. (2015).

\subsection{Profiles for dark matter only haloes}

Dark matter haloes in $\Lambda$ CDM are fairly well described by the NFW (Navarro et al. 1997) double power-law profile. While power laws are robust for understanding and are analytically friendly to work with, it has been made apparent that dark matter density profiles are not perfectly captured by the power-law construction. Navarro et al. $(2004,2010)$ demonstrated that higher resolution dark matter density profiles have log-slopes ${ }^{5}$ that decrease monotonically as $r$ approaches the centre, which is not captured by the NFW at small $r$. This indicates that the innermost regions of CDM haloes are shallower than an NFW. Their study suggested a different radial profile for DMO haloes, starting with the log-slope relation:

$\frac{\mathrm{d} \log \rho}{\mathrm{d} \log r}(r)=-2\left(\frac{r}{r_{-2}}\right)^{\alpha_{\epsilon}}$.

This results in the three-parameter Einasto profile:

$\log \left[\frac{\rho_{\text {Ein }}(r)}{\rho_{-2}}\right]=-\frac{2}{\alpha_{\epsilon}}\left[\left(\frac{r}{r_{-2}}\right)^{\alpha_{\epsilon}}-1\right]$,

where $\alpha_{\epsilon}$ is the so-called shape parameter that tunes how slow or fast the slope changes with radius, and $r_{-2}$ (as well as $\rho_{-2}:=\rho\left(r_{-2}\right)$ ) is the radius (density) at which the logarithmic slope of the density profile is equal to -2 , i.e. $\mathrm{d} \log \rho /\left.\mathrm{d} \log r\right|_{r=r_{-2}}=-2$.

The shape parameter, $\alpha_{\epsilon}$, is a key component of equation (4). When obtained from Einasto profile fits to dark matter haloes of cosmological simulations, it has been shown to correlate with the overdensity peak height of the dark matter halo and is calibrated based on the cosmology (e.g. Gao et al. 2008; Dutton \& Macciò 2014; Klypin et al. 2016). Fixing $\alpha_{\epsilon} \simeq 0.16$ has been shown to provide a good fit for DMO haloes throughout the literature (Merritt et al. 2006; Prada et al. 2006; Gao et al. 2008). With this choice, $\rho_{\text {Ein }}$ becomes a two-parameter function, one that still provides a better fit to DMO simulations than the two-parameter NFW profile. ${ }^{6}$ Recently, Wang et al. (2019) have shown that the two-parameter version of $\rho_{\text {Ein }}$ provides a adequate fit for DMO haloes over 30 orders of magnitude in halo mass. We fix $\alpha_{\epsilon}=0.16$ in what follows.

\subsection{Cored profile for feedback-affected CDM haloes}

We follow Navarro et al. (2004) and consider the behaviour of the log-slope of the density profiles for our galaxy haloes as a function of radius. Fig. 3 shows log-slope profiles for four classifications of haloes in our full-physics runs: 'cusps', 'small cores', 'large cores', and 'MW-mass haloes'. The haloes simulated with FIRE-2 physics are plotted as the coloured solid curves, while their respective DMO analogues are shown as the dashed lines with the same colour. Starting with the upper left-hand panel, low-mass dwarfs tend to be hosted by cuspy dark matter haloes. Similarly, haloes with small cores tend to host higher mass classical dwarfs. Haloes with the largest cores correspond the brightest dwarf galaxies, which we have seen previously in Fig 2, while MW-mass galaxies have dark matter halo profiles that are more complicated (and are discussed further next). For reference, the solid black line shows the log-slope of the

\footnotetext{
${ }^{5}$ We refer 'log-slope' as the logarithmic derivative of the local density profile: $\mathrm{d} \log \rho / \mathrm{d} \log r$.

${ }^{6}$ Of course, one can acquire even better density profile fits to as good as $5-10$ per cent for haloes in our mass range when leaving $\alpha_{\epsilon}$ as a free parameter, as this value tailors to each shape to the dark matter halo. This, however, leaves ambiguity in the value of $r_{-2}$, as this is now dependent on $\alpha_{\epsilon}$.
} 

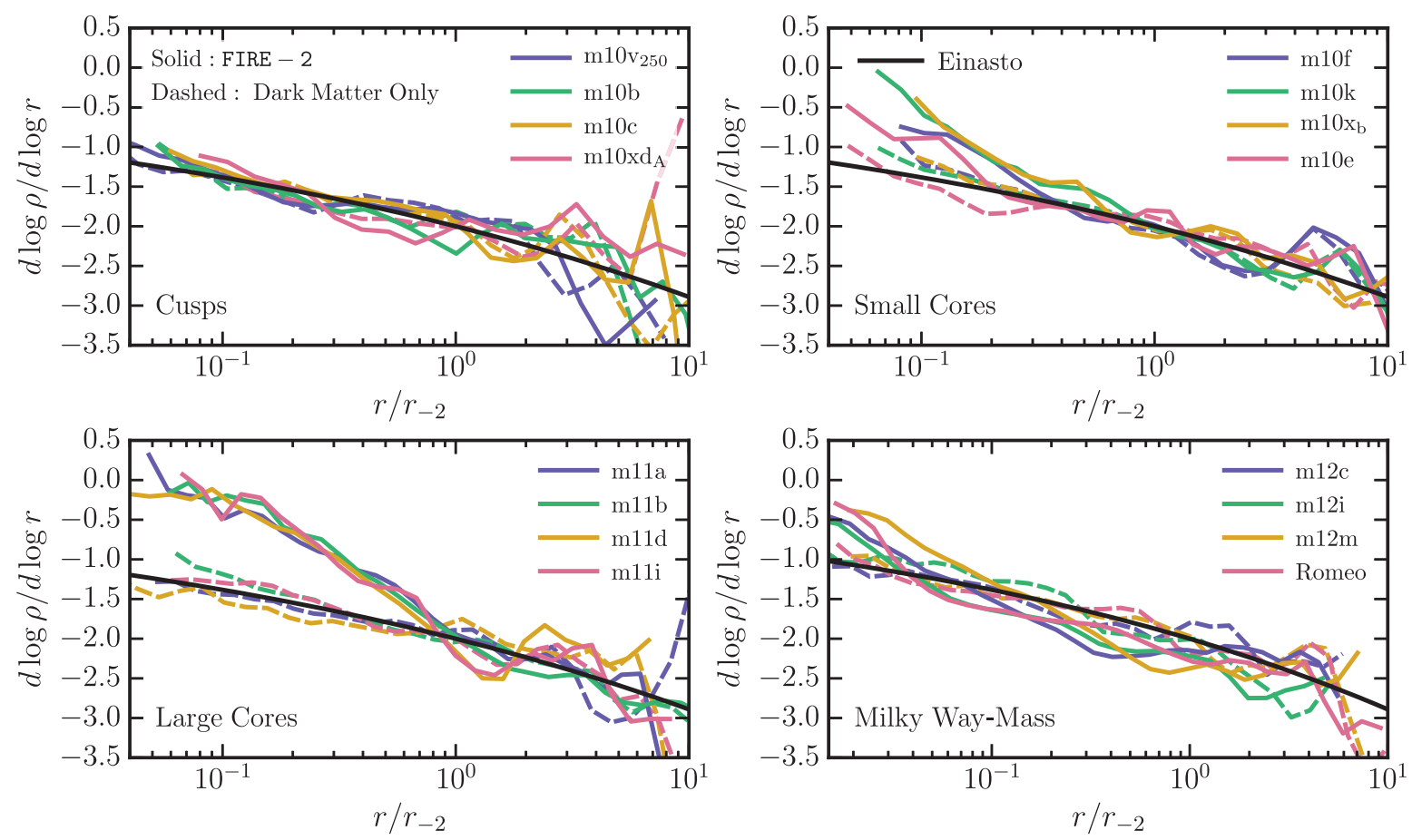

Figure 3. Comparison of the log-slope behaviour. The four panels show galaxies grouped by the behaviour of their inner density profiles: galaxies with cusps, small cores, large cores, and MW-mass haloes. The resolved portions of the FIRE-2 galaxies are depicted as the solid lines, while the resolved DMO analogue profiles are plotted as the dashed lines. The solid black line illustrates the slope expected from equation (3). All of the radial values are normalized by $r_{-2}$ of the DMO analogues, which are computed by fitting equation (4) to each individual dashed curves. As expected, the galaxies with cusps are well described by equation (4). Galaxies with small cores have profiles that start to rise very slowly towards $\operatorname{dlog} \rho / \operatorname{dog} r=0$ at $\sim r_{-2}$. The largest cores in our sample are seen to have slight excesses in the density at around $r_{-2}$ (the 'dip' in the profile) and begins to rise substantially for decreasing values of $r$. Milky Way-mass haloes are the outliers in the trend, in which the galaxies' log-slopes are inconsistent with their dark matter analogues beginning at $r_{-2}$. At radii $r \ll r_{-2}$, the log-slopes are shown to form cores abruptly.

Einasto profile, equation (3). The galaxies and DMO analogues have their radii normalized by $r_{-2}$ from the DMO runs.

As expected, equation (3) captures the log-slope trend of the DMO haloes. The same is true for FIRE-2 runs with low stellar mass fraction ('cusps' in this case). Haloes labelled 'small cores' tend to slightly deviate from equation (3), with upturns in the log-slope trend for $r \lesssim 0.03 \times r_{-2}$. The lower left-hand panel contains galaxy haloes (the solid lines) that approach $\operatorname{dlog} \rho / \operatorname{dlog} r=0$ at small radii - that is, a true core. This behaviour never occurs beyond $r_{-2}$ of the analogous DMO profiles, and cores are only see at $r \ll r_{-2}$. MWmass haloes have more complicated profiles. Their log-slopes tend to lie below the log-slope of DMO analogues from $r \simeq[0.1-1] \times$ $r_{-2}$; this is a consequence of baryonic contraction. However, we see that at $r \ll r_{-2}$, the log-slopes begin to rise towards 0 , indicating that small cores can form in our MW sample.

To capture the behaviour illustrated in Fig. 3, we start by writing a more general form of equation (3) that allows the log-slope to increase more sharply within a physical core radius, $r_{\mathrm{c}}$ :

$$
\frac{\mathrm{d} \log \rho}{\mathrm{d} \log r}(r)=-2\left(\frac{r}{\tilde{r}_{\mathrm{s}}}\right)^{\alpha_{\epsilon}} \widetilde{\mathcal{C}}\left(r \mid r_{\mathrm{c}}\right) .
$$

Implemented here is a radially dependent damping function, $\widetilde{C}\left(r \mid r_{\mathrm{c}}\right)$, which is designed to control the rate of which the profile dampens within $r_{\mathrm{c}}$. The variable $\tilde{r}_{\mathrm{s}}$ plays a similar role as $r_{-2}$ in equation (3), but will no longer be the radius where the log-slope is equal to -2 owing to the presence of $r_{\mathrm{c}}$. We demand that the behaviour of the damping function satisfies the limiting cases of $\widetilde{C} \rightarrow 1$ and $\tilde{r}_{\mathrm{s}} \rightarrow r_{-2}$ as $r_{\mathrm{c}} \rightarrow 0$ to (i) capture the qualitative expectations of cores that can substantially vary in size and (ii) revert back to the form of $\rho_{\operatorname{Ein}}$ in the absence of a core.

We adopt the following form:

$\widetilde{\mathcal{C}}\left(r \mid r_{\mathrm{c}}\right)=\left(1+\frac{r_{\mathrm{c}}}{r}\right)^{\alpha_{\epsilon}-1}$,

such that

$\frac{\mathrm{d} \log \rho}{\mathrm{d} \log r}(r)=-2\left(\frac{r}{\tilde{r}_{\mathrm{s}}}\right)^{\alpha_{\epsilon}}\left(1+\frac{r_{\mathrm{c}}}{r}\right)^{\alpha_{\epsilon}-1}$.

In particular, the log-slope of the density profile approaches zero more quickly for larger values of $r_{\mathrm{c}}$. Integrating out equation (7) gives us a cored counterpart of $\rho_{\text {Ein }}$, the core-Einasto profile:

$\log \left[\frac{\rho_{\mathrm{cEin}}(r)}{\tilde{\rho}_{\mathrm{s}}}\right]=-\frac{2}{\alpha_{\epsilon}}\left[\left(\frac{r+r_{\mathrm{c}}}{\tilde{r}_{\mathrm{s}}}\right)^{\alpha_{\epsilon}}-1\right]$.

Here, $\tilde{\rho}_{\mathrm{s}}$ is a density-free parameter in the fit. In what follows we set $\alpha_{\epsilon}=0.16$, which reduces the expression to a three-parameter profile. In the limiting case of $r_{\mathrm{c}} \rightarrow 0$, we re-acquire $\rho_{\text {Ein }}$, where now $\tilde{\rho}_{\mathrm{s}} \rightarrow \rho_{-2}$. Note that the central density with the presence of a core, $\rho_{0}:=\rho_{\text {cEin }}(r=0)$, is parametrized as

$\rho_{0}=\tilde{\rho}_{\mathrm{s}} \exp \left\{-\frac{2}{\alpha_{\epsilon}}\left[\left(\frac{r_{\mathrm{c}}}{\tilde{r}_{\mathrm{s}}}\right)^{\alpha_{\epsilon}}-1\right]\right\}$.

Alternatively, we can reparametrize $\tilde{\rho}_{\mathrm{s}}$ by mapping to $\tilde{\rho}_{-2}:=$ $\rho_{\text {cEin }}\left(r_{-2}\right)$, the density (and radius) where the log-slope is equal to 
-2 . This allows us to re-express equation (8) as

$\log \left[\frac{\rho_{\mathrm{cEin}}(r)}{\tilde{\rho}_{-2}}\right]=-\frac{2}{\alpha_{\epsilon}}\left[\left(\frac{r+r_{\mathrm{c}}}{\tilde{r}_{\mathrm{s}}}\right)^{\alpha_{\epsilon}}-\left(\frac{r_{-2}+r_{\mathrm{c}}}{\tilde{r}_{\mathrm{s}}}\right)^{\alpha_{\epsilon}}\right]$,

which certainly work in our zero core limit to re-acquire equation (4). However, this expression now introduces an additional free parameter, $r_{-2}$, which can likely lead to degenerate results in acquiring $r_{\mathrm{c}}$ and $\tilde{r}_{\mathrm{s}}$. With that, we prefer to adopt the form of equation (8) for our analysis hereinafter. Analytic expressions for the mass profile, gravitational potential, and energy for the core-Einasto profile are presented in Appendix B.

\subsection{Resulting profile fits}

All functional fits are performed using the Levenberg-Marquart minimization algorithm. We restrict our radial density profile fits to the radial range of $r_{\text {conv }}$ to $r_{\text {vir }}$. Best-fitting models are obtained by simultaneously adjusting the parameters of the analytical density profiles to minimize a figure of merit function, defined by

$Q^{2}=\frac{1}{N_{\text {bins }}} \sum_{i}^{N_{\text {bins }}}\left[\log _{10} \rho_{i}-\log _{10} \rho_{i}^{\text {model }}\right]^{2}$,

which weights all the logarithmic radial bins equally and, for a given radial range, is fairly independent of the number of bins used (Navarro et al. 2010). That is, the minimum figure of merit, denoted as $Q_{\min }$, quantifies the residuals of the true profile from the model caused by shape differences induced in the fitting routine.

\subsubsection{Local dark matter density}

Fig. 4 provides example fits for a sample of dark matter density profiles. Dark matter haloes simulated using FIRE-2 (the black curves) are fitted with $\rho_{\text {cEin }}$ (pink dashed), while the DMO analogues (the grey line) are fitted with $\rho_{\text {Ein }}$ (dashed green). In each panel, we list the galaxy's stellar mass fraction $\left(M_{\star} / M_{\text {halo }}\right)$, stellar mass $\left(M_{\star}\right)$, dark matter core radius $\left(r_{\mathrm{c}}\right)$ given by fitting $\rho_{\text {cEin }}$, and the goodness of fit $\left(Q_{\text {min }}\right)$ from fitting $\rho_{\text {cEin }}$. The location of the best-fitting dark matter core radius, scaled by the virial radius, is indicated by the black arrow in each panel. Table E2 lists the fit results for all of our galaxies, including the fit parameters and the $Q_{\min }$ values. We can see that the value $r_{\mathrm{c}}$ is effectively determined for a wide range of galaxy sizes. For even the worst profile fits (e.g. m10xh with $Q_{\min }=$ 0.074; top right-hand panel), the value of $r_{\mathrm{c}}$ is still identified at the location where one's eye might pick out a dark matter core in the local density profile.

As a way of examining the robustness of equation (8), we fit core-Einasto to the DMO analogues and found that in every case the best-fitting core-radii were either zero or smaller than the radius of convergence. This provides confidence that this profile does not force or impose cores that do not exist in the resolved regions of the halo. However, it does suggest that $r_{\mathrm{c}}$ values smaller than the convergence limit should not be taken as robust indications for the existence of real cores. For example, the upper left-hand panel of Fig. 4 shows an $\rho_{\text {cEin }}$ fit to $\mathrm{m}_{10 \mathrm{v}_{250}}$ (baryon simulated), a profile that is unaltered by feedback in the resolved region owing to its small stellar mass. The best-fitting core radius $\left(r_{\mathrm{c}} \simeq 50 \mathrm{pc}\right)$ is much smaller than the radius of convergence ( $r_{\text {conv }} \simeq 160 \mathrm{pc}$ ) in this case.

While we find success in characterizing dwarf galaxies with $\rho_{\text {cEin }}$, almost all of the MW-mass haloes have cored regions that are more sharply pronounced than enabled by the $\rho_{\text {cEin }}$ profile. As one can see (e.g. m12b and Romeo), the values of $r_{\mathrm{c}}$ from the fits do not coincide with the locations of the bend seen in the simulated profiles. ${ }^{7}$ Based on our entire sample of MW-mass haloes, we find that the $\rho_{\text {cEin }}$ profile performs less well for MW-mass haloes that have both a small central dark matter core and baryonic contraction in the inner densities. On the other hand, MW-mass haloes with little evidence of either baryonic contraction (e.g. m12z) or a core are successfully characterized by $\rho_{\text {cEin }}$. MW-mass haloes with no core, but with only baryonic contraction, are also well modelled by $\rho_{\text {Ein }}$. In Appendix C, we formulate a more general core profile with one additional free parameter that captures the behaviour for baryonic contracted haloes with cores. This allows us to accurately quantify the core radii for the rest of our MW-mass haloes.

\subsubsection{Density profile residuals}

Profile residuals of the local dark matter density are presented in Fig. 5 for DMO analogue fitted with the Einasto profile (left) and to the dark matter haloes of the FIRE-2 physics runs fit to core-Einasto (right). Results are split into the four galaxy classifications defined in Section 2. The residuals for the left and right columns are comparable, which is remarkable given that the right-hand fits have only one additional free parameter to account for the full impact of complex galaxy formation physics. Notice that the largest deviations are present large radii $\left(r \gtrsim 0.3 r_{\text {vir }}\right)$. This behaviour has been seen in the past for DMO haloes, where the outer regions may not be fully relaxed (e.g. Ludlow et al. 2010, 2016), and may contain large substructures.

While we have only two ultrafaint galaxies (the blue curves) in our sample, both galaxies are well described to 10 per cent for a majority of the radii. This is unsurprising, as these haloes lack the requisite star formation to induce cores; the core-Einasto fit is therefore effectively the same as a standard Einasto fit, with $r_{\mathrm{c}}$ values that are smaller than the convergence radius. Almost all of the classical dwarf galaxies (the green curves) have excellent core-Einasto fits, with deviations in the range $10-15$ per cent at worst. At small radii $(r \lesssim 0.1 \times$ $\left.r_{\text {vir }}\right)$, core-Einasto is shown to be sufficient in fitting the FIRE-2 haloes compared to their DMO analogues in the same radial regions. For a majority of the brightest dwarfs in our sample, deviations are constrained within 15 per cent. For MW-mass haloes, the quality of the fit can range from quite good to as bad as 20 per cent. As mentioned previously, the worst fits are for the MW-mass haloes impacted by both baryonic contraction and feedback-induced core formation at small radii. We find deviations of $10-15$ per cent in the innermost regions for profiles of MWs with just cores (e.g. m12z in Fig. 4) or just having baryonic contraction with no cores.

In both columns, there are are hints of a sinusoidal feature in the residuals. This behaviour is not unusual when simplified fits are compared to detailed dark matter halo profiles (e.g Griffen et al. 2016). Reducing the residual behaviour even more would require more free parameters in the form of $\widetilde{\mathcal{C}}$ in equation (5) and/or allowing the value of $\alpha_{\epsilon}$ to vary from halo-to-halo. However, given that the gross residuals for our core-Einasto fits to the FIRE-2 runs are close to those of Einasto fits to DMO runs, we are satisfied that the given parametrization provides a useful balance between simplicity and accuracy. In Appendix D, we do find that our haloes are modelled better by the three-parameter core-Einasto profile than two alternative three-parameter profiles: the core extension for the NFW from Peñarrubia et al. (2012) and Dekel + from Dekel et al. (2017) and Freundlich et al. (2020).

\footnotetext{
${ }^{7}$ The core radius of Romeo from the $\rho_{\text {cEin }}$ fit does not appear in Fig. 4 (bottom right-hand panel) since the fitted value of $r_{\mathrm{c}}$ is located inside the region of numerical convergence $\left(r_{\mathrm{c}} / r_{\mathrm{vir}}<10^{-3}\right)$.
} 


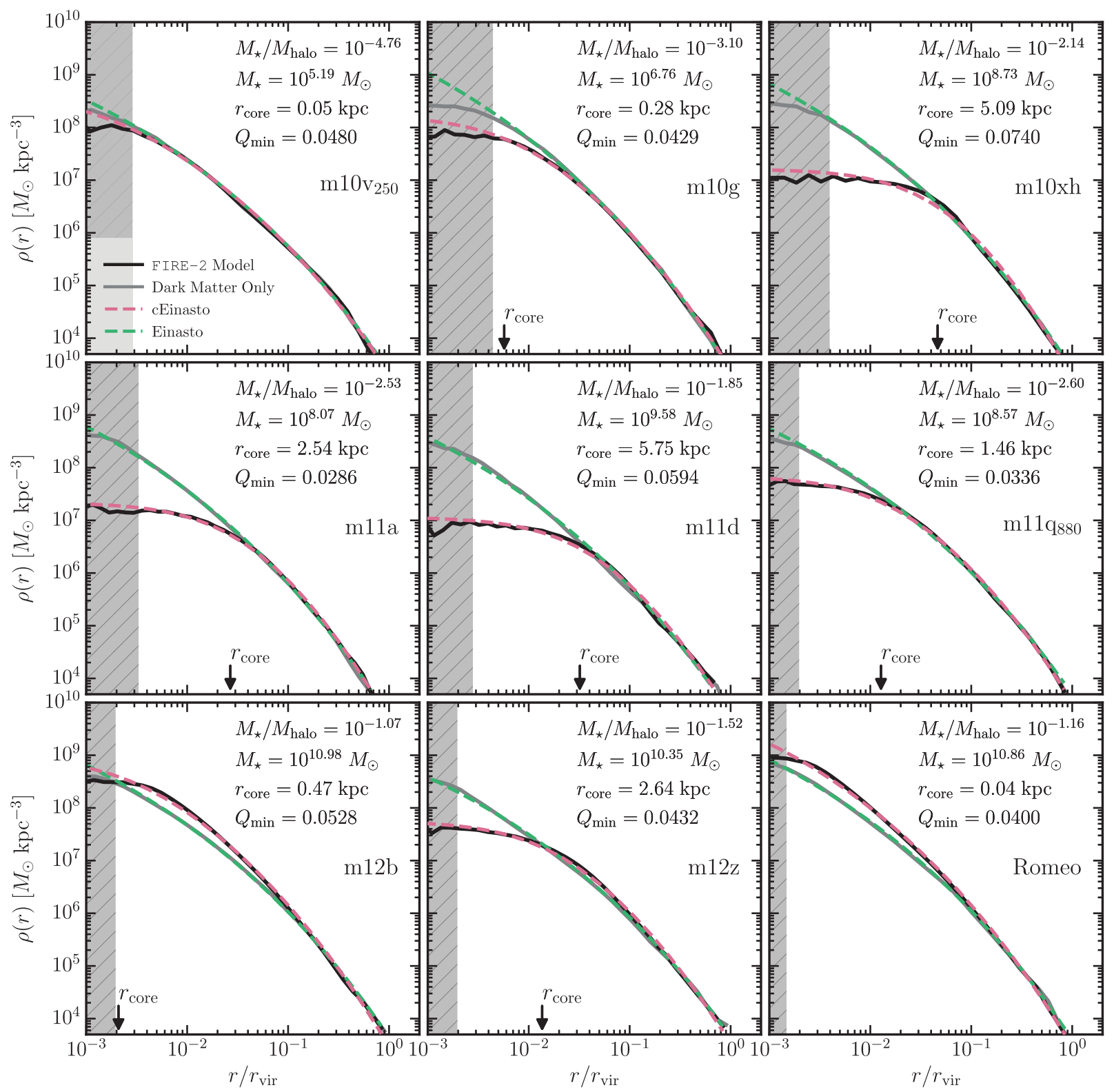

Figure 4. Profiles of the local dark matter density: The $\rho_{\text {cEin }}$ fits (the pink-dashed curves, with $\alpha_{\epsilon}=0.16$ ) are plotted along with the FIRE-2 galaxies (the black curves) for a sample of galaxy haloes. The $\rho_{\text {Ein }}$ fits (the green-dashed curves, with $\alpha_{\epsilon}=0.16$ ) to the density profiles of DMO analogues (the grey curves) are plotted as well. The vertical grey band encloses the radius where numerical two-body relaxation might effect the halo. Each panel has a list of relevant parameters for each galaxy: the stellar mass fraction $\left(M_{\star} / M_{\text {halo }}\right)$, the stellar mass $\left(M_{\star}\right)$, the dark matter core radius from the $\rho_{\mathrm{cEin}}$ profile fit $\left(r_{\mathrm{c}}\right)$, and the minimum value of the merit function $\left(Q_{\mathrm{min}}\right)$ that indicates the goodness of fit. The fitted dark matter core radius, $r_{\mathrm{c}}$, is indicated by the black arrow pointing along the radial axis to show its location in units of $r_{\text {vir }}$. For most of the depicted galaxies, the $\rho_{\text {cEin }}$ profile fits perform exceptionally well in parametrizing the location of $r_{\mathrm{c}}$. Note that these examples include the full range of fit quality in our sample (as measured by $Q_{\min }$ ), including some of the poorest fits, e.g. m10xh in the upper right corner.

\subsubsection{Dark matter circular velocity}

Fig. 6 provides an alternative view of the results shown in Fig. 4: it shows the circular velocity curves of the dark matter component, ${ }^{8}$ $V_{\text {circ }}(r)=\sqrt{G M(<r) / r}$, for the same haloes presented in Fig. 4, each normalized by $V_{\max }:=\max \left[V_{\text {circ }}(r)\right]$ of the dark matter

${ }^{8}$ For the analysis of observed galaxies, spherically averaged rotation curves are typically presented using their total mass, i.e. their combined baryonic and dark matter components. We chose to show just the dark matter components here to compare with our core-Einasto model. curve. The analytical profiles for $V_{\text {cEin }}$ and $V_{\text {Ein }}$ are plotted using equations (B5) and (B6), respectively, for the values obtained from the fits shown in Fig. 4. These analytical curves are normalized by the $V_{\max }$ values of the simulated haloes to which they are fitted. For profile fits overestimating (or underestimating) the mass found in the simulated profiles by $15-20$ per cent (e.g. m10xh and m11d), the most substantial effects can seen at the outer radii, near where $V_{\max }$ is attained. However, even for the worst profile fits in our sample, the central density normalization is well-captured for dwarf galaxies of varying stellar mass fractions. 

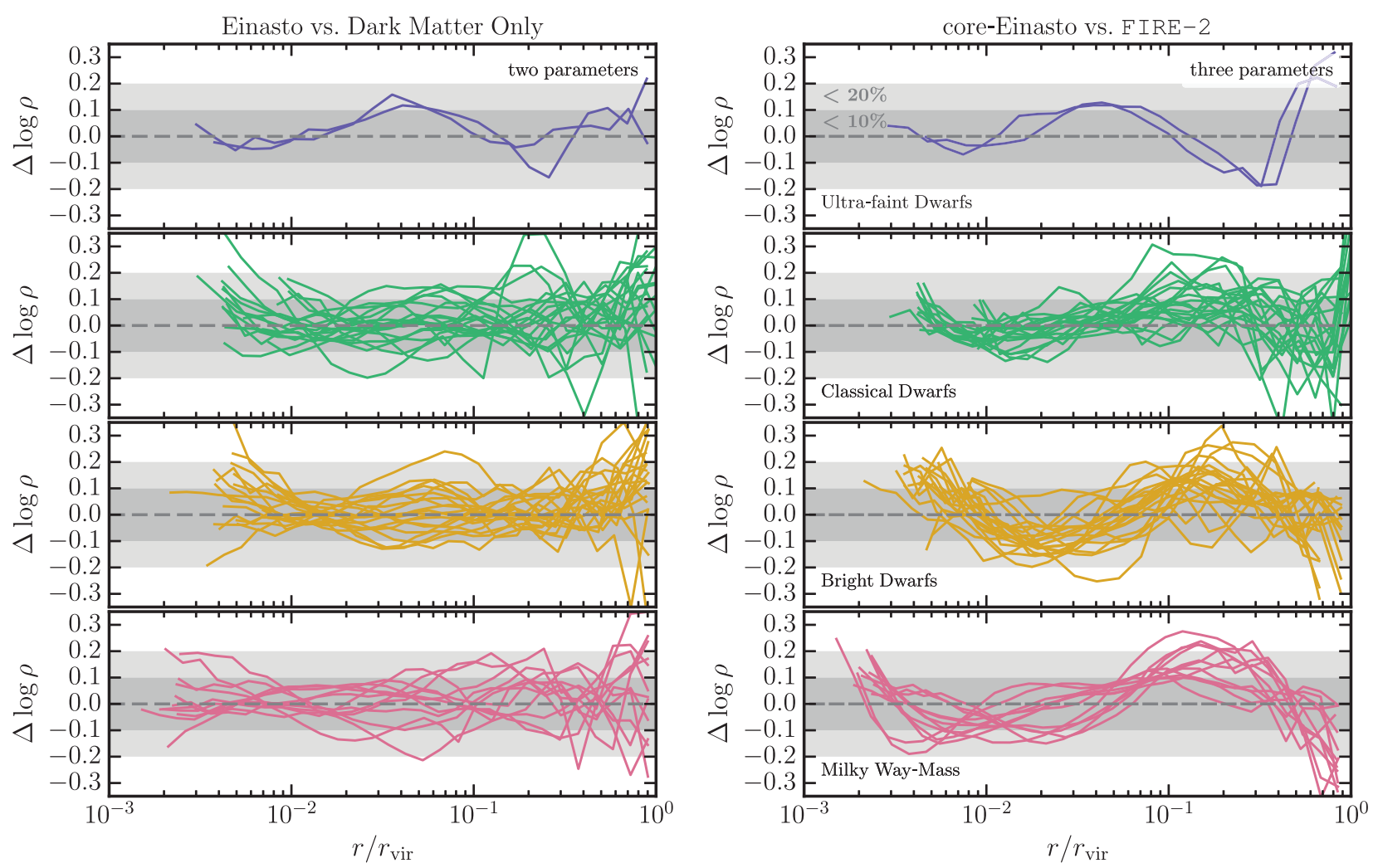

Figure 5. Profile residuals: Deviation from the best profile fits for each individual halo (fit subtracted from simulation). The left column shows residuals for fits to our DMO analogues using Einasto profiles with $\alpha_{\epsilon}=0.16$. The right column shows residuals for the hydrodynamic simulations of the same haloes fit using the core-Einasto profile with $\alpha_{\epsilon}=0.16$. For clarity, we have grouped haloes by the four classification groups discussed in Section 2 in each row: ultrafaint dwarfs, classical dwarfs, bright dwarfs, and MW-mass haloes. Residuals are computed from the inner-most resolved radius, $r_{\text {conv }}$, out the virial radius of each halo. The darker and lighter shaded grey enclose residuals of 10 per cent and 20 per cent, respectively. The core-Einasto fits to the full physics runs are almost as good as the Einasto fits are for the DMO haloes. The offsets are less than 15 per cent in the inner regions of classical dwarfs and most bright dwarfs. Several of MW-size haloes show worse fits, with offsets as large as 20 per cent, which is a result of both baryonic contraction and feedback-induced dark matter cores.

\subsection{Parametrization of the physical core radius}

For the left plot in Fig. 7, we show the relationship between $M_{\star} / M_{\text {halo }}$ and the fitted values of $r_{\mathrm{c}}$. The circular points denote the values of $r_{\mathrm{c}}$ that we verify as resolved cores (with $r_{\mathrm{c}}>r_{\mathrm{conv}}$ for the local dark matter density profiles). This sample includes the MWmass core radii fit using using the four parameter function $\rho_{\text {cEin, } \mathrm{BC}}$ (the cyan highlights) described in Appendix $\mathrm{C}$ instead of their $r_{\mathrm{c}}$ values from $\rho_{\text {cEin }}$ (shown by the grey points for reference). The squares denote best-fitting core radii that have values smaller the numerical convergence region $\left(r_{\mathrm{c}}<r_{\text {conv }}\right)$. It is important to note that in some cases, we obtain fit values of $r_{\mathrm{c}}$ that are formally smaller than $r_{\text {conv }}$ yet large enough that the halo is not well described by the standard $\rho_{\text {Ein }}$ form. This comes about because dark matter haloes impacted by stellar feedback produce dark matter profiles that are no longer self-similar in nature, meaning the core-Einasto fit balances $\tilde{r}_{\mathrm{s}}$ and $r_{\mathrm{c}}$ to accommodate the shape of the density profile.

We see that our robustly determined $r_{\mathrm{c}}$ values $\left(r_{\mathrm{c}}>r_{\text {conv }}\right)$, begin to appear at the higher mass end for the classical dwarf galaxy regime, $M_{\star} / M_{\text {halo }} \gtrsim 7 \times 10^{-3}$, with values that are physically quite small, $r_{\mathrm{c}} \simeq 0.2-0.3 \mathrm{kpc}$. As the stellar mass fraction increases toward the region of bright dwarf galaxies, $M_{\star} / M_{\text {halo }} \simeq 10^{-3}-10^{-2}$, the sizes of the core radii, $r_{\mathrm{c}}$, increase with $M_{\star} / M_{\text {halo }}$. Importantly, the largest dark matter cores, $r_{\mathrm{c}} \simeq 5-6 \mathrm{kpc}$, coincide with the stellar mass fraction at the peak core formation that we have seen previously $\left(M_{\star} / M_{\text {halo }}\right.$ $\simeq 5 \times 10^{-3}$ ). A majority of the galaxies at the MW-mass scale have dark matter cores as $r_{\mathrm{c}} \simeq 1-2 \mathrm{kpc}$, though two remain fairly cuspy ( $\mathrm{m} 12 \mathrm{r}$ and $\mathrm{m} 12 \mathrm{w})$. To provide further insight into observations of real galaxies comparable to the simulations analysed here, the right plot in Fig. 7 shows the trend of $r_{\mathrm{c}}$ with $M_{\star}$. The largest cores tend to form in galaxies with $M_{\star} \simeq 10^{8-9} \mathrm{M}_{\odot}$. Notably, a significant amount of scatter is seen for fixed value of $r_{\mathrm{c}} \simeq 2-3 \mathrm{kpc}$, which tends to be apparent for galaxies with $M_{\star} \simeq 10^{8-11} \mathrm{M}_{\odot}$.

The formation of small cores for MW-mass haloes using FIRE-1 was discussed in Chan et al. (2015), where they found that small cores for MW-size galaxies tend form in the low-mass galaxy progenitors at $z \sim 2$, which have stellar-to-halo mass ratios suitable for core formation. These progenitors have their resulting innermost dark matter profile amplified at $z=0$ due to baryonic contraction. This phenomena also drives out old stars formed in situ in MWlike galaxies (El-Badry et al. 2018b). Other simulation groups have not reported the existence of small cores at the MW-mass regime. This could, however, be due to differences in numerical resolution. For example, the NIHAO simulations presented in Tollet et al. (2016) and Macciò et al. (2020) study MW-mass haloes at a lower resolution than ours, with convergence down to $r_{\text {conv }} \simeq 1.25 \mathrm{kpc}$ compared 330-500 pc in our runs (see Table E1). The MW cores in our simulations are $\sim 1 \mathrm{kpc}$ in size. Such cores would be difficult to form without having a convergence radius smaller than this limit. 


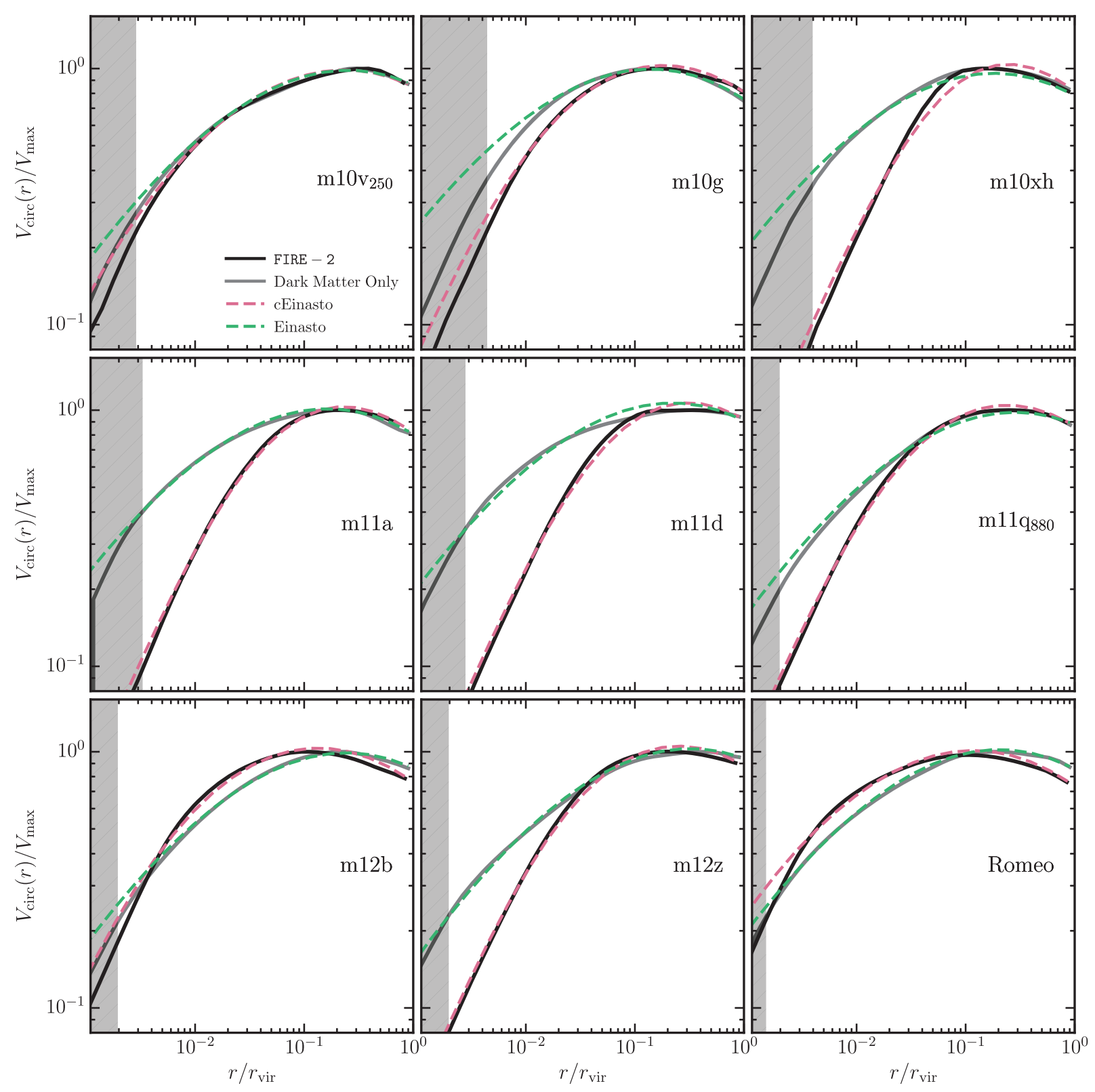

Figure 6. Dark matter circular velocity curves. Shown are the dark matter components of the circular velocity curves, $V_{\text {circ }}(r)=\sqrt{G M(<r) / r}$, of the same haloes presented in Fig. 4. The dashed pink and green curves are plotted using the analytical forms of equations (B5) and (B6), respectively. Curves of $V_{\text {cEin }}$ and $V_{\text {Ein }}$ are normalized by $V_{\max }$ of the galaxy and DMO analogue, respectively. Analytical fits are able to capture the density normalization of the simulated haloes robustly for all of the dwarf galaxies, even while it can underestimate or overestimate the integrated mass in at the outer radii.

We find that the relationship between $r_{\mathrm{c}}$ and $x=M_{\star} / M_{\text {halo }}$ (and $x=M_{\star} / \mathrm{M}_{\odot}$ ) can be captured as a double power law

$r_{\mathrm{c}}(x)=10^{\mathcal{A}_{1}}\left(\mathcal{A}_{2}+\frac{x}{x_{1}^{*}}\right)^{-\beta_{1}}\left(\frac{x}{x_{2}^{*}}\right)^{\gamma_{1}} \mathrm{kpc}$,

where $\left\{\beta_{1}, \gamma_{1}\right\}$ are the free parameter slopes that control the transition of $x$. The quantities $\left\{x_{1}^{*}, x_{2}^{*}\right\}$ are normalization parameters associated with both slopes, and $\left\{\mathcal{A}_{1}, \mathcal{A}_{2}\right\}$ are constants of the fit. Best-fitting parameters for $x=M_{\star} / M_{\text {halo }}$ and $M_{\star} / M_{\odot}$ are given in Table 1 . The trend for our plotted data for $r_{\mathrm{c}}$ as a function of $M_{\star} / M_{\text {halo }}$ and $M_{\star} / M_{\odot}$ is shown by the blue curves in the left and right plots in Fig. 7, respectively.

Fig. 8 is similar to left plot in Fig. 7 except with the values of $r_{\mathrm{c}}$ normalized by the size of the dark matter halo virial radius $\left(r_{\mathrm{vir}}\right.$; left plot $)$ or the half-stellar-mass radius of the galaxy it hosts $\left(r_{1 / 2}\right.$; right plot) as a function of $M_{\star} / M_{\text {halo }}$. Notably, the normalization for each plot roughly follows the same trend that we have seen in previous figures: as $M_{\star} / M_{\text {halo }}$ increases from $10^{-4}$ to $10^{-2}$, galaxies have larger cores, even relative to the size of the dark matter halo or its central galaxy. The trend peaks at the mass scale of robust core formation. At this peak, the brightest galaxies tend have cores of $r_{\mathrm{c}} \sim 0.04 r_{\mathrm{vir}}$ (albeit with large scatter) and $r_{\mathrm{c}} \sim r_{1 / 2}$. Interestingly, most MW-mass

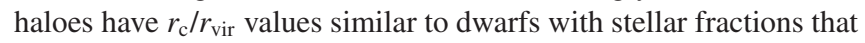
are 100 times lower and $r_{\mathrm{c}} / r_{1 / 2}$ values comparable to many of the brightest dwarfs.

\subsection{Parametrization of $\tilde{\boldsymbol{r}}_{\mathrm{s}}$}

We wish to quantify how the free parameter, $\tilde{r}_{\mathrm{s}}$, is related to $r_{-2}$ from using $\rho_{\text {cEin }}$, the radius at which the log-slope of the local 

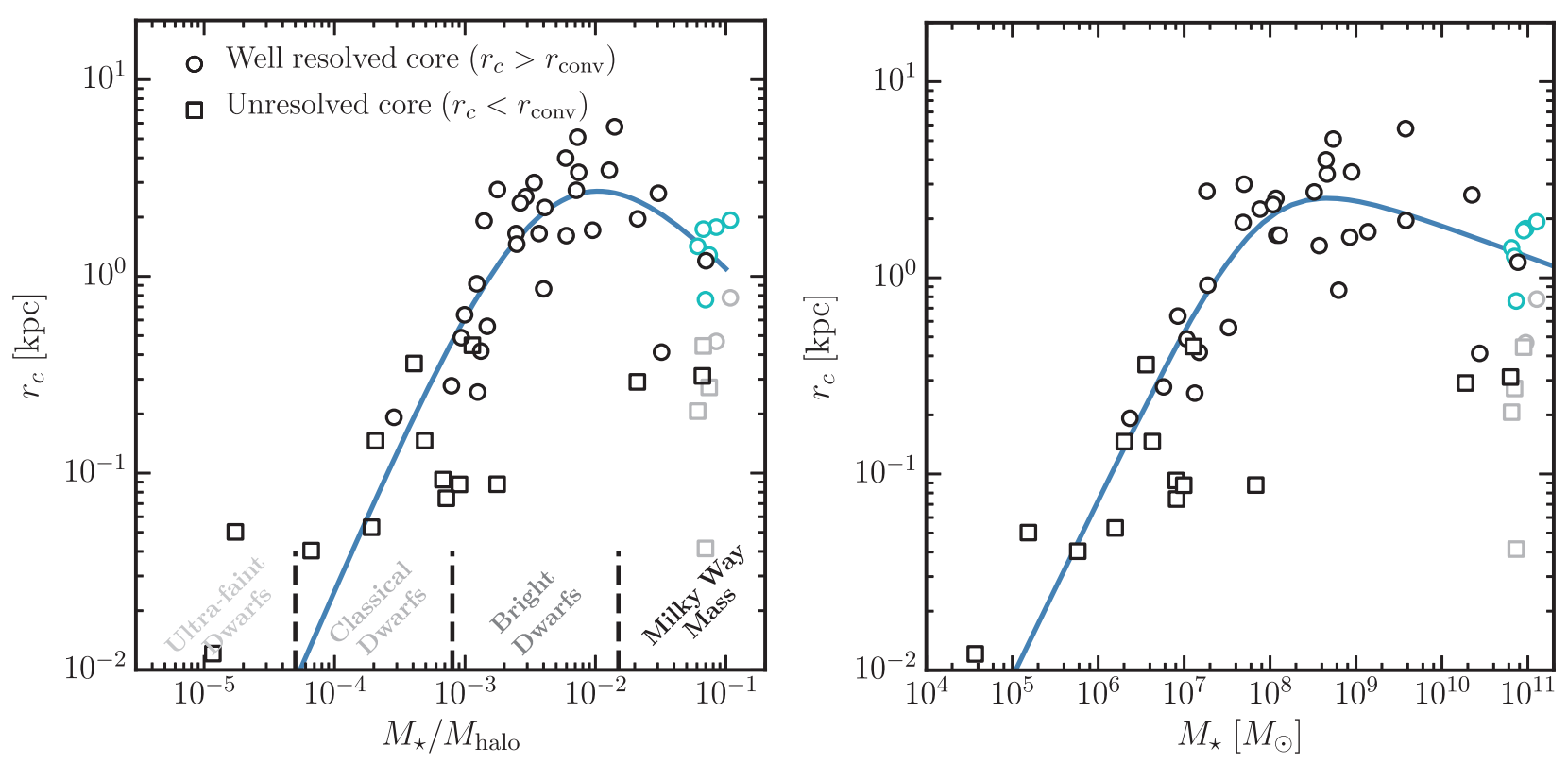

Figure 7. Feedback-induced core formation. The circles show core radii that are larger than the convergence radius of the simulation $\left(r_{\mathrm{c}}>r_{\mathrm{conv}}\right)$, while the squares are values smaller than the convergence radius $\left(r_{\mathrm{c}}<r_{\text {conv }}\right)$. MW haloes with significant baryonic contraction, which are therefore not as well fit by the $\rho_{\text {cEin }}$ function, are shown in light grey. The cyan points show $r_{\mathrm{c}}$ values for MW-mass galaxies returned from a four-parameter 'baryonic contracted cored-Einasto' profile, $\rho_{\mathrm{cEin}, \mathrm{BC}}$, introduced in Appendix C, to better account for baryonic contraction. Left: Core radius as a function of stellar to halo mass ratio. The solid blue curve is a fit to the dark black and cyan points using equation (12), with the best-fitting parameters given in Table 1. We note that this trend mirrors results shown in Fig. 1, with the largest core radii values occuring in the 'Bright Dwarfs' regime. Right: Dark matter core radius as a function of $M_{\star}$. Peak core formation, while scattered, appears around $M_{\star}=10^{8-9} \mathrm{M}_{\odot}$. The solid blue curve is our best fitting line using equation (12) and best-fitting parameters from Table 1 for $x=M_{\star}$.

Table 1. Best-fitting parameters for the physical core radius, $r_{\text {core }}$. For complete data set: $-3.54 \lesssim \log _{10}\left(M_{\star} / M_{\text {halo }}\right) \lesssim-0.97$ and $6.37 \lesssim \log _{10}\left(M_{\star} / \mathrm{M}_{\odot}\right)$ $\lesssim 11.10$

\begin{tabular}{lcccccc}
\hline Parameter & $\mathcal{A}_{1}$ & $\mathcal{A}_{2}$ & $x_{1}^{*}$ & $x_{2}^{*}$ & $\beta_{1}$ & $\gamma_{1}$ \\
\hline$M_{\star} / M_{\text {halo }}$ & 1.21 & 0.71 & $7.2 \times 10^{-3}$ & 0.011 & 2.31 & 1.55 \\
$M_{\star} / \mathrm{M}_{\odot}$ & 1.33 & $4.3 \times 10^{7}$ & 1.93 & 0.55 & 1.06 & 0.90
\end{tabular}

Note. Use equation (12) for either $x=M_{\star} / M_{\text {halo }}$ or $x=M_{\star} / \mathrm{M}_{\odot}$.

dark matter density is equal to -2 , in the presence of a dark matter core. Unfortunately, the relation between $\tilde{r}_{\mathrm{s}}$ and $r_{-2}$ for the FIRE-2 dark matter haloes cannot be solved analytically as the additional power of $\alpha_{\epsilon}$ means they are non-linearly related. However, we can paramtrize the covariance between $\tilde{r}_{\mathrm{s}}$ and $r_{-2}$ from introducing $r_{\mathrm{c}}$. Fig. 9 shows the ratio of $\tilde{r}_{\mathrm{s}}$ to $r_{-2}$ as a function of $M_{\star} / M_{\text {halo }}$ for the FIRE-2 haloes. Here, $r_{-2}$ is interpolated from only the $\rho_{\text {cEin }}$ fits. As expected, dwarf galaxies with no cores (or cores small enough to effectively be approximated as $r_{\mathrm{c}}=0$ ) have $\tilde{r}_{\mathrm{s}} \simeq r_{-2}$. As we transition towards the region of peak core formation, $\tilde{r}_{\mathrm{s}}$ gradually decreases relative to $r_{-2}$. We then see a sudden upturn at the MWmass scale, which is a consequence of baryonic contraction. The relation for $\tilde{r}_{\mathrm{s}}$ to $r_{-2}$ as a function of $M_{\star}$ is also discussed in Appendix A.

The relationship between $\tilde{r}_{\mathrm{s}} / r_{-2}$ and either $x=M_{\star} / M_{\text {halo }}$ (or $x=$ $\left.M_{\star} / \mathrm{M}_{\odot}\right)$ can be captured as a double power law:

$\left[\tilde{r}_{\mathrm{s}} / r_{-2}\right](x)=\left(1+\frac{x}{x_{3}^{*}}\right)^{-\beta_{2}}+\mathcal{B}\left(\frac{x}{x_{4}^{*}}\right)^{\gamma_{2}}$,

where $\left\{\beta_{2}, \gamma_{2}\right\}$ are the free parameter slopes that control the transition, the quantities $\left\{x_{3}^{*}, x_{4}^{*}\right\}$ are the normalization values associated with these slopes, and $\mathcal{B}$ is a constant. The best-fitting parameters for
$x=M_{\star} / M_{\text {halo }}$ are given in Table 2. The trend for our data is plotted as the blue curve in Fig. 9.

\subsection{Parametrization of the halo concentration}

The stellar feedback in dark matter haloes also affects the halo concentration through the gravitational coupling of dark matter to the rapidly changing central gravitational potential. We adopt the halo concentration parameter $c_{\mathrm{vir}}:=r_{\mathrm{vir}} / r_{-2}$. This definition of $c_{\mathrm{vir}}$ will be applied for the established results modelled by $\rho_{\text {cEin }}, \rho_{\text {cEin, BC }}$, and $\rho_{\text {Ein }} .{ }^{9}$ Ratios of the concentration parameter between the FIRE-2 haloes, $c_{\mathrm{F} 2}$, and their DMO analogues, $c_{\mathrm{DM}}$, are shown in the lefthand panel of Fig. 10 as a function of $M_{\star} / M_{\text {halo }}$. The result from Di Cintio et al. (2014b) is plotted as the pink curve. We also extend this discussion with the parametrization done for $M_{\star}$ in Appendix A.

Galaxies with lower stellar mass fraction limit $\left(M_{\star} / M_{\text {halo }} \lesssim\right.$ $10^{-4}$ ) have values of $c_{\text {vir }}$ comparable to their DMO analogues. Noticeable differences of the concentrations become apparent as $M_{\star} / M_{\text {halo }}$ starts to increase towards the classical dwarf and bright galaxy regime. Importantly, as $M_{\star} / M_{\text {halo }}$ approaches the peak of sufficient core formation, the halo concentrations for the FIRE-2 galaxies are conspicuously smaller - by $30-50$ per cent - than the halo concentrations of their DMO analogues. This could mean that the strength of stellar feedback, which we can also probe by the size $r_{\mathrm{c}}$, in these haloes has been strong enough to affect the density structure out to $r_{-2}$, an effect not seen previously (e.g. compare with the pink curve from Di Cintio et al. 2014b). However, the

${ }^{9}$ For the FIRE-2 haloes fitted well with $\rho_{\text {cEin }}$ and the MWs fitted with $\rho_{\text {cEin, BC }}$ in Appendix $\mathrm{C}$, the value of $r_{-2}$ is interpolated from the analytical profile fits, while for the DMO haloes, $r_{-2}$ is taken from the free parameter fit of $\rho_{\text {Ein }}$. 

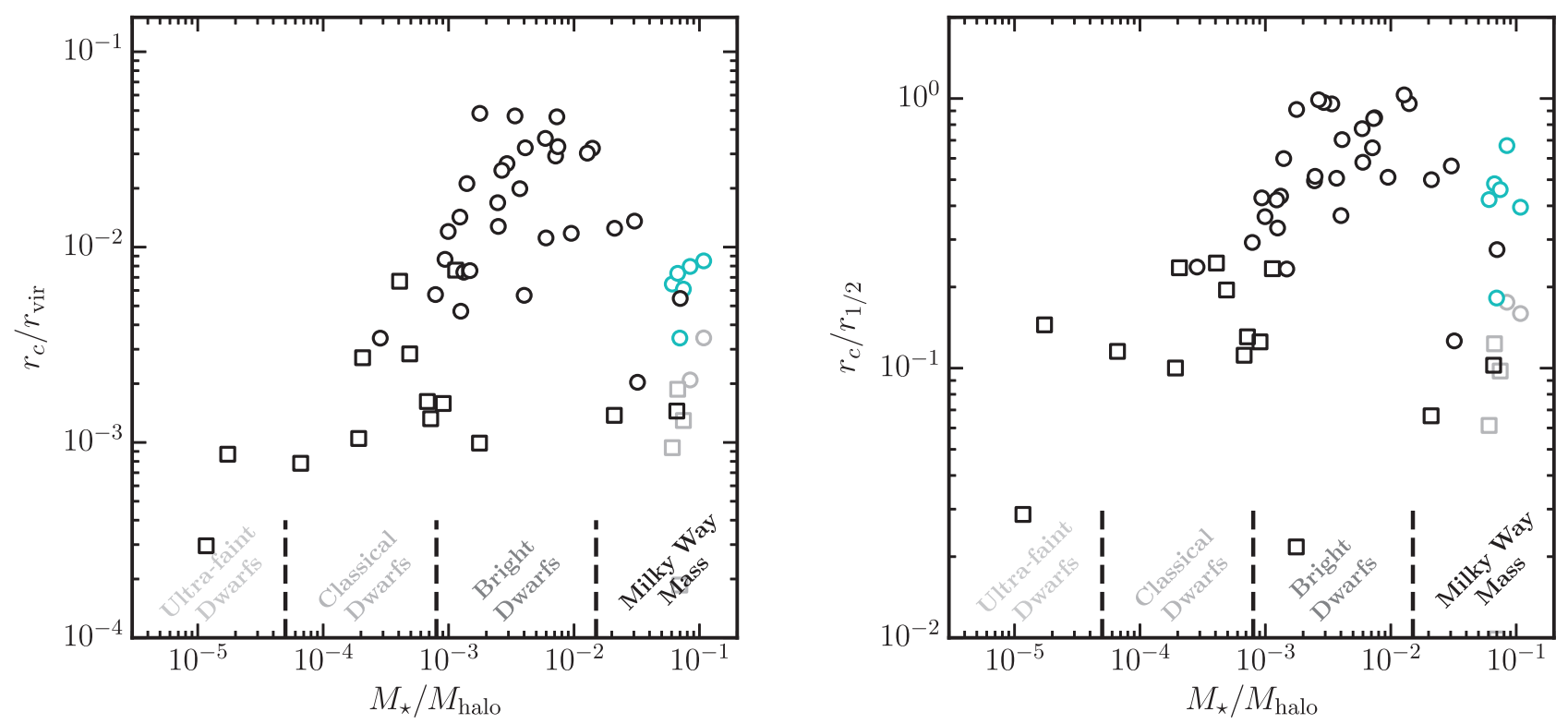

Figure 8. Core radius relative to the halo and galaxy size. Similar to Fig. 7, except with the core radii scaled by the virial radius of the dark matter haloes (left) and stellar half-mass radius of the galaxies (right). Left: The fractional size of cores rises toward the regime of peak core formation, where $r_{\mathrm{c}} \simeq 0.05 r_{\mathrm{vir}}$. MW-mass haloes have $r_{\mathrm{c}} / r_{\mathrm{vir}}$ values comparable to those of dwarf galaxies with $M_{\star} / M_{\text {halo }} \sim 10^{-3}$. Right: All resolved cores are constrained to a lower bound of $r_{\mathrm{c}} \gtrsim 0.1 r_{1 / 2}$. At peak core formation, $r_{\mathrm{c}} \simeq r_{1 / 2}$ for some of the the brightest dwarfs.

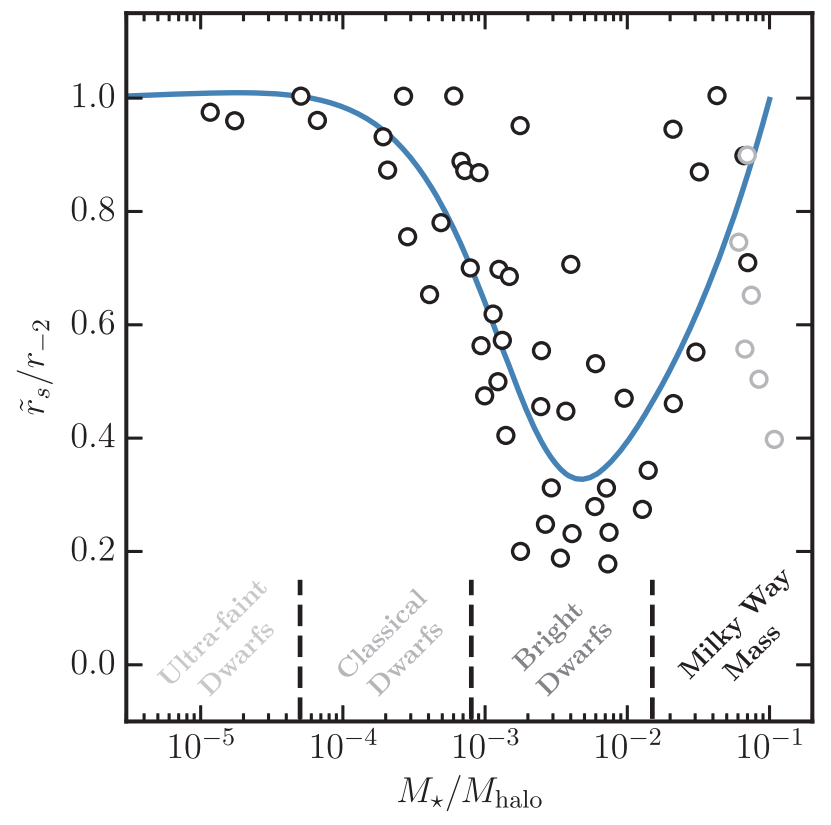

Figure 9. Effects of core formation on the global structure of the dark matter halo. A trend can be seen between the fitting parameter, $\tilde{r}_{\mathrm{s}}$, and the interpolated scale radius, $r_{-2}$, from the $\rho_{\text {cEin }}$ profile. We again show the results not well fitted with $\rho_{\text {cEin }}$, which are highlighted in light grey like in Fig 7. As the core radius of the haloes becomes larger, i.e. as we increase $M_{\star} / M_{\text {halo }} \sim 5 \times 10^{-3}$, the physical interpretation of $\tilde{r}_{\mathrm{S}}$ changes. This result shows that the formation of a core (found most prominently in the regime of the bright dwarfs) results in a change to the dark matter halo at larger scales (as parametrized by $r_{-2}$ ). The solid blue curve is our best fits using equation (13).

relation from Di Cintio et al. (2014b) used the parameters obtained from fitting the $\alpha \beta \gamma$-profile to acquire $r_{-2}$, while we numerically interpolated from our resulting profile fits. We explore the differences in concentration that arise for the same haloes when fitting different
Table 2. Best-fitting parameters for $\tilde{r}_{\mathrm{s}} / r_{-2}$.

\begin{tabular}{lccccc}
\hline Parameter & $\mathcal{B}$ & $x_{3}^{*}$ & $x_{4}^{*}$ & $\beta_{2}$ & $\gamma_{2}$ \\
\hline$M_{\star} / M_{\text {halo }}$ & 1.51 & 0.044 & 0.28 & 31.79 & 0.40 \\
$M_{\star} / \mathrm{M}_{\odot}$ & 0.098 & $5.1 \times 10^{6}$ & $1.4 \times 10^{6}$ & 0.57 & 0.20 \\
\hline
\end{tabular}

Note. Use equation (13) for either $x=M_{\star} / M_{\text {halo }}$ or $x=M_{\star} / \mathrm{M}_{\odot}$.

profiles in Appendix D. We find that when using the $\alpha \beta \gamma$-profile, the concentration can shift somewhat, but there is a tendency to be lower in the bright dwarf regime, following a similar qualitative trend shown in Fig. 10.

As stellar fractions reach the the MW regime, we see the opposite effect: the concentrations of our galaxy haloes are significantly larger than their DMO analogues because of baryonic contraction.

The relationship between the concentration parameters of our galaxy haloes can be parametrized as a double power law:

$\left[c_{\mathrm{F} 2} / c_{\mathrm{DM}}\right](x)=\left(1+\frac{x}{x_{5}^{*}}\right)^{-\beta_{3}}+\mathcal{C}\left(\frac{x}{x_{5}^{*}}\right)^{\gamma_{3}}$,

where either $x=M_{\star} / M_{\text {halo }}$ or $x=M_{\star} / \mathrm{M}_{\odot},\left\{\beta_{3}, \gamma_{3}\right\}$ are slopes, and $x_{5}^{*}$ is a free normalization value to anchor the transition between slopes, and $\mathcal{C}$ is a constant. Best-fitting parameters for $x=M_{\star} / M_{\text {halo }}$ are given in Table 3. The trend for our data is plotted as the blue curve in the left plot of Fig. 10.

The right plot in Fig. 10 shows the dark matter halo concentration directly: $c_{\text {vir }}$ as a function of the dark matter halo mass, $M_{\text {halo }}$. The black-filled circles are the results for the FIRE-2 haloes, while the open circles are the DMO analogues. The solid green curve traces the recent results of the concentration-mass relation from Wang et al. (2019), which extends to masses all way down to the Earth mass dark matter haloes. Note that Wang et al. (2019) uses the same concentration definition as we do as well. Additionally, they also fit haloes with an Einasto profile same shape parameter we adopted ( $\alpha_{\epsilon}=0.16$ ). The DMO analogues in our halo mass range follow the Wang et al. (2019) relation with significant scatter about the 

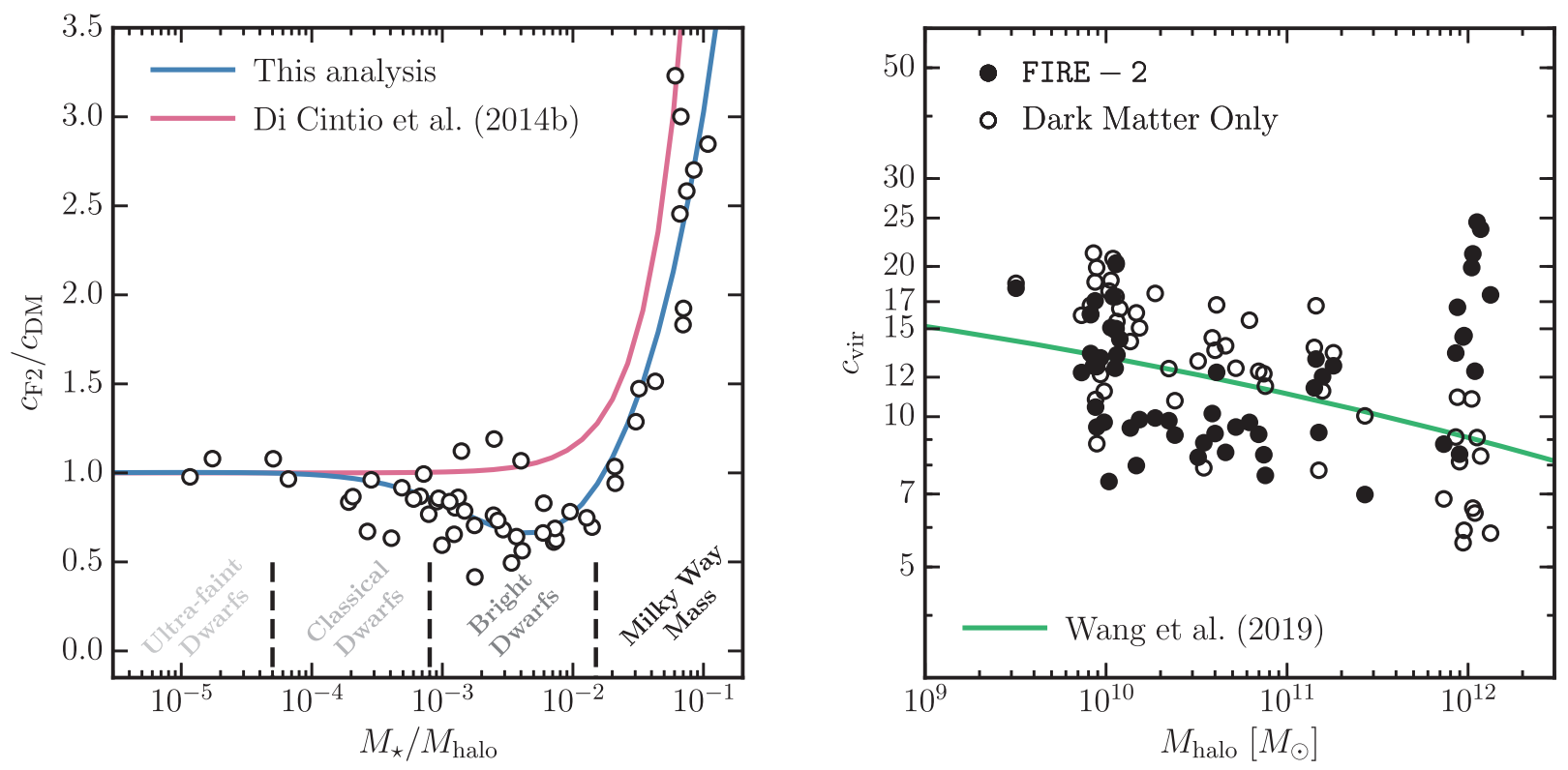

Figure 10. The impact of feedback on halo concentration. Halo concentration defined by $c_{\mathrm{vir}}:=r_{\mathrm{vir}} / r_{-2}$. Left: FIRE-2 halo concentrations $\left(c_{\mathrm{F} 2}\right)$ using $r_{-2}$

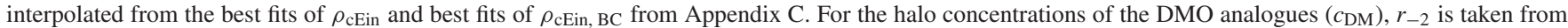
the free parameter fit of $\rho_{\text {Ein. }}$. Galaxies with have the largest cores (the brightest dwarfs) have their halo concentrations lowered by a factor of two compared to DMO analogue concentrations. The solid blue curve is our best fit of the scatter points using equation (14). Also plotted is the fit from Di Cintio et al. (2014b) as the solid pink curve for comparison. Right: Concentration as a function of dark matter halo mass. Galaxies and DMO analogues are denoted by the filled and unfilled black circles, respectively. The solid green curve is the concentration relation from Wang et al. (2019), which was also calibrated using the $c_{\text {vir }}$ from the Einasto profile (including the same shape considered here, $\alpha_{\epsilon}=0.16$ ).

Table 3. Best-fitting parameters for $c_{\mathrm{F} 2} / c_{\mathrm{DM}}$.

\begin{tabular}{lcccc}
\hline Parameter & $\mathcal{C}$ & $x_{5}^{*}$ & $\beta_{3}$ & $\gamma_{3}$ \\
\hline$M_{\star} / M_{\text {halo }}$ & 0.374 & $4.28 \times 10^{-3}$ & 1.80 & 0.66 \\
$M_{\star} / \mathrm{M}_{\odot}$ & $6.39 \times 10^{-4}$ & $1.77 \times 10^{5}$ & 0.057 & 0.62 \\
\hline
\end{tabular}

Note. Use equation (14) for either $x=M_{\star} / M_{\text {halo }}$ or $x=M_{\star} / \mathrm{M}_{\odot}$.

median. Interestingly, galaxy haloes with $M_{\text {halo }}=10^{10-11} \mathrm{M}_{\odot}$ all have about the same concentrations of $c_{\text {vir }} \simeq 9$, with small scatter. In the $M_{\text {halo }}=10^{12} \mathrm{M}_{\odot}$ region, baryonic contraction of the galaxy can increase the halo concentration significantly, to $c_{\mathrm{vir}} \simeq 15-25$ ). Observational measurements of the MW's halo concentration, which usually assume an NFW profile, have often found values typical of those we find here for our FIRE-2 haloes $\left(c_{\mathrm{vir}} \approx 15-25\right)$ - well above the expectation for DMO haloes of that mass $\left(c_{\mathrm{vir}} \sim 9\right.$; Battaglia et al. 2005; Catena \& Ullio 2010; Deason et al. 2012; Nesti \& Salucci 2013). This then also suggests that for real galaxies, the predictions from Wang et al. (2019) will be an underestimated.

\section{SUMMARY AND CONCLUDING REMARKS}

In this paper, we studied and modelled the $z=0$ dark matter density profiles of 54 zoom-in galaxy simulations run using the FIRE-2 feedback model. Our sample includes galaxies with stellar masses ranging from ultrafaint dwarfs to MW-mass galaxies, a factor of around seven decades in stellar mass and three decades in halo mass. Details on these simulated haloes, as well as parameter fits for each dark matter halo, are provided in Appendix E.

The most significant contribution of this paper has been the introduction of the 'core-Einasto': a new, three-parameter analytic density profile that provides a good fit to our FIRE-2 galaxy haloes by allowing for a prominent constant density core, equation (8) Specifically, our main conclusions are as follows:

(i) We find that feedback creates prominent cores in the centres of dark matter haloes that have galaxy stellar masses $M_{\star} / M_{\text {halo }} \simeq$ $5 \times 10^{-3}$ or $M_{\star} \sim 10^{9} \mathrm{M}_{\odot}$, roughly comparable to the stellar masses spanning the mass ranges of the SMC and the LMC (Figs 2, 6, and 7). This mass regime is in agreement with previous studies (e.g. Di Cintio et al. 2014a; Tollet et al. 2016). Feedback-induced core formation becomes less important for galaxies with larger and smaller stellar masses.

(ii) We find no evidence that feedback alters the density structure of haloes that host galaxies smaller than $M_{\star} \simeq 10^{6} \mathrm{M}_{\odot}$ or $M_{\star} / M_{\text {halo }} \lesssim$ $10^{-4}$ down to radii $\sim 0.5$ per cent of $r_{\text {vir }}(\sim 100$ pc; see also Fitts et al. 2017). This in turn results in concentration values matching those seen in DMO analogues (Fig. 10). However, in FIRE-2 simulations with higher resolution, feedback may produce cores $\sim 100 \mathrm{pc}$ in such galaxies (see Wheeler et al. 2019).

(iii) The core-Einasto profile, equation (8), takes the Einasto profile, equation (4), and adds one additional parameter, a core radius $r_{\mathrm{c}}$. The profile returns to the standard Einasto form as $r_{\mathrm{c}} \rightarrow 0$. With a fixed $\alpha_{\epsilon}=0.16$, we find that the three-parameter core-Einasto profile is able to characterize the majority of our feedback-impacted dark matter haloes almost as well as the standard two-parameter Einasto profile does for DMO haloes (Figs 4-5). In Appendix D, we compare fits using the core-Einasto profile to two other threeparameter profiles (core-NFW and Dekel + ) and show that the coreEinasto provides a better fit to FIRE-2 haloes.

(iv) Fitted core radii are the largest $\left(r_{\mathrm{c}} \simeq 1-5 \mathrm{kpc}\right)$ for bright dwarf galaxies of $M_{\star} / M_{\text {halo }} \simeq 5 \times 10^{-3}$ (or $M_{\star} \sim 10^{9} \mathrm{M}_{\odot}$; Fig. 7). Fitted core radii become smaller as the stellar-to-halo mass ratio moves away from this value (or equivalently, at both higher and lower stellar masses). The physical core radius is found to never be much larger 
than the stellar half-light radius, $r_{\mathrm{c}} \lesssim r_{1 / 2}$, and only approaches $r_{1 / 2}$ in galaxies of the characteristic mass for core formation, $M_{\star} \sim 10^{9} \mathrm{M}_{\odot}$ (Fig. 8)

(v) Feedback and galaxy formation alters the global structure of dark matter haloes well beyond the core region (Figs 9-10). Haloes that host bright dwarf galaxies are often less concentrated than their DMO analogues, with $c_{\text {vir }}$ values 30 per cent smaller. This differs slightly from the results in Di Cintio et al. (2014b), who found no change in concentration at this mass scale. At higher masses, approaching the MW scale, the trend reverses and haloes become much more concentrated owing to baryonic contraction.

(vi) While baryonic contraction makes haloes more concentrated and denser at the stellar half-light radius for MW size galaxies, we find that feedback can still produce small dark matter cores of $\sim 0.5-2 \mathrm{kpc}$ in size at this mass scale. The formation of cores in MW-size haloes was previously discussed in Chan et al. (2015). The combination of core-formation and baryonic contraction makes the resultant profiles complicated enough that equation (8) does less well at capturing the full shape (with $\approx 20$ per cent residuals, Fig. 5 ). To accommodate these features, we introduce a four-parameter contracted core profile in Appendix C (see Fig. C1). The presence of dark matter cores in MW-size galaxies might be supported by dynamical modelling of MW data. Portail et al. (2017) find evidence for a dark matter core comparable in size to what we quantify our feedback-affected MW-mass haloes.

Though our results for core-Einasto and $r_{\mathrm{c}}$ relations have focused on haloes at $z=0$, the evolution of $r_{\mathrm{c}}$ throughout cosmic time would provide an interesting future avenue of study, one that could provide further insight on the energy budget needed to transform cusps to cores in $\triangle \mathrm{CDM}$ throughout cosmic time. Similarly, the methodology implemented and discussed in our analysis may be beneficial for a variety of studies in galaxy formation with alternative dark matter models. That is, our methods can be applicable in constraining characteristics of dark matter haloes formed in other dark matter models. For example, dwarf galaxies simulated in selfinteracting dark matter have characteristic central densities that are proportional to the interaction cross-section (see Rocha et al. 2013). Preliminary results indicate that cores in self-interacting dark matter haloes are 'sharper' than those in feedback-affected CDM haloes, perhaps indicating a path for differentiating between the two models in the presence of exquisite data (Straight et al., in preparation).

Perhaps the most exciting direction for future work will involve direct comparisons and modelling of observational data. To enable comparisons with observations, we provide fitting functions for $r_{\mathrm{c}}$ and other profile fit parameters as a function of $M_{\star} / M_{\text {halo }}$ (see equations (12-14) and Tables 1-3). Appendix A provides fits as a function of $M_{\star}$. Best-fitting parameters for all 54 of our galaxies are listed in Table E1. Resulting core-Einasto parameters can be utilized with analytic expressions for the mass profile, gravitational potential, and energy as presented in Appendix B.

We have also shown that the dark matter rotation curves are well captured by the core-Einasto fits in our simulations in Fig. 6, which motivates a comparison to current rotation curve data, such as the that from the THINGS survey (Walter et al. 2008; Oh et al. 2015) or SPARC (Lelli, McGaugh \& Schombert 2016). For examples of modelling with analytical profiles, we refer to the reader to analysis conducted by, but not limited to, Kamada et al. (2017), Katz et al. (2017), Ren et al. (2019), Kaplinghat, Ren \& Yu (2019), Robles, Bullock \& Boylan-Kolchin (2019), and Li et al. (2020). With the advent of future astrometric data being collected by Gaia (Gaia
Collaboration 2016a,b, 2018a,b), our model can also be combined with the central density normalizations obtainable in Lazar \& Bullock (2020) from the proper motions of dispersion-supported galaxies to constrain possible core radii and central densities via equation (9).

\section{ACKNOWLEDGEMENTS}

This article was worked to completion during the COVID-19 lockdown and would not have been possible without the labors of our essential workers. We would like to thank the referee for helpful comments on the earlier versions of this article. AL and JSB was supported by the National Science Foundation (NSF) grant AST-1910965. MBK acknowledges support from NSF CAREER award AST-1752913, NSF grant AST-1910346, NASA grant NNX17AG29G, and HST-AR-14282, HST-AR-14554, HST-AR15006, HST-GO-14191, and HST-GO-15658 from the Space Telescope Science Institute, which is operated by AURA, Inc., under NASA contract NAS5-26555. TKC is supported by STFC astronomy consolidated grant ST/T000244. ASG is supported by the McDonald Observatory at the University of Texas at Austin, through the Harlan J. Smith fellowship. AW received support from NASA through ATP grant 80NSSC18K1097 and HST grants GO-14734, AR-15057, AR15809, and GO-15902 from STScI; the Heising-Simons Foundation; and a fellowship from the Hellman Foundation. KE is supported by an NSF graduate research fellowship. Support for CW was provided by NASA through the NASA Hubble Fellowship grant \#10938 awarded by STScI. DK acknowledges support from NSF grant AST-1715101 and the Cottrell Scholar Award from the Research Corporation for Science Advancement. Simulations presented in this work utilized resources granted by the Extreme Science and Engineering Discovery Environment (XSEDE), which is supported by NSF grant OCI-1053575. CAFG was supported by NSF through grants AST1517491, AST-1715216, and CAREER award AST-1652522; by NASA through grant 17-ATP17-0067; and by a Cottrell Scholar Award from the Research Corporation for Science Advancement. The analysis in this paper depended on the PYTHON packages NUMPY (van der Walt, Colbert \& Varoquaux 2011), SCIPY (Oliphant 2007), and MATPLOTLIB (Hunter 2007); We are thankful to the developers of these tools. This research has made intensive use of NASA's Astrophysics Data System (http://ui.adsabs.harvard.edu/) and the arXiv eprint service (http://arxiv.org).

\section{DATA AVAILABILITY}

The data supporting the plots within this article are available on reasonable request to the corresponding author. A public version of the GIZMO code is available at http://www.tapir.caltech.edu/ phopkin s/Site/GIZMO.html. Additional data including simulation snapshots, initial conditions, and derived data products are available at https: //fire.northwestern.edu/data/.

\section{REFERENCES}

Battaglia G. et al., 2005, MNRAS, 364, 433

Behroozi P. S., Wechsler R. H., Wu H.-Y., 2013, ApJ, 762, 109

Behroozi P., Wechsler R. H., Hearin A. P., Conroy C., 2019, MNRAS, 488, 3143

Benítez-Llambay A., Frenk C. S., Ludlow A. D., Navarro J. F., 2019, MNRAS, 488, 2387

Binney J., Tremaine S., 2008, Galactic Dynamics, 2nd edn, Princeton University Press, Princeton, New Jersey 
Blumenthal G. R., Faber S. M., Flores R., Primack J. R., 1986, ApJ, 301, 27

Bose S. et al., 2019, MNRAS, 486, 4790

Boylan-Kolchin M., Bullock J. S., Kaplinghat M., 2011, MNRAS, 415, L40

Brook C. B., Di Cintio A., 2015, MNRAS, 450, 3920

Brook C. B., Stinson G., Gibson B. K., Wadsley J., Quinn T., 2012, MNRAS, 424, 1275

Brooks A. M., Zolotov A., 2014, ApJ, 786, 87

Bryan G. L., Norman M. L., 1998, ApJ, 495, 80

Bullock J. S., Boylan-Kolchin M., 2017, ARA\&A, 55, 343

Catena R., Ullio P., 2010, JCAP, 2010, 004

Chan T. K., Kereš D., Oñorbe J., Hopkins P. F., Muratov A. L., FaucherGiguère C. A., Quataert E., 2015, MNRAS, 454, 2981

Chan T. K., Kereš D., Wetzel A., Hopkins P. F., Faucher-Giguère C.-A., ElBadry K., Garrison-Kimmel S., Boylan-Kolchin M., 2018, MNRAS, 478, 906

Cole D. R., Dehnen W., Wilkinson M. I., 2011, MNRAS, 416, 1118

Deason A. J., Belokurov V., Evans N. W., An J., 2012, MNRAS, 424, L44

Dekel A., Ishai G., Dutton A. A., Maccio A. V., 2017, MNRAS, 468, 1005

Di Cintio A., Brook C. B., Macciò A. V., Stinson G. S., Knebe A., Dutton A. A., Wadsley J., 2014a, MNRAS, 437, 415

Di Cintio A., Brook C. B., Dutton A. A., Macciò A. V., Stinson G. S., Knebe A., 2014b, MNRAS, 441, 2986

Dubinski J., Carlberg R. G., 1991, ApJ, 378, 496

Dutton A. A., Macciò A. V., 2014, MNRAS, 441, 3359

Dutton A. A., Macciò A. V., Buck T., Dixon K. L., Blank M., Obreja A., 2019, MNRAS, 486, 655

Einasto J., 1965, Tr. Astrofizicheskogo Inst. Alma-Ata, 5, 87

El-Badry K., Wetzel A., Geha M., Hopkins P. F., Kereš D., Chan T. K., Faucher-Giguère C.-A., 2016, ApJ, 820, 131

El-Badry K. et al., 2018a, MNRAS, 473, 1930

El-Badry K. et al., 2018b, MNRAS, 480, 652

El-Zant A., Shlosman I., Hoffman Y., 2001, ApJ, 560, 636

Faucher-Giguère C.-A., Lidz A., Zaldarriaga M., Hernquist L., 2009, ApJ, 703,1416

Ferland G. J., Korista K. T., Verner D. A., Ferguson J. W., Kingdon J. B., Verner E. M., 1998, PASP, 110, 761

Fitts A. et al., 2017, MNRAS, 471, 3547

Flores R. A., Primack J. R., 1994, ApJ, 427, L1

Freundlich J. et al., 2020, preprint (arXiv:2004.08395)

Gaia Collaboration, 2016a, A\&A, 595, A1

Gaia Collaboration, 2016b, A\&A, 595, A2

Gaia Collaboration, 2018a, A\&A, 616, A1

Gaia Collaboration, 2018b, A\&A, 616, A12

Gao L., Navarro J. F., Cole S., Frenk C. S., White S. D. M., Springel V., Jenkins A., Neto A. F., 2008, MNRAS, 387, 536

Garrison-Kimmel S., Rocha M., Boylan-Kolchin M., Bullock J. S., Lally J., 2013, MNRAS, 433, 3539

Garrison-Kimmel S., Boylan-Kolchin M., Bullock J. S., Kirby E. N., 2014, MNRAS, 444, 222

Garrison-Kimmel S., Bullock J. S., Boylan-Kolchin M., Bardwell E., 2017, MNRAS, 464, 3108

Garrison-Kimmel S. et al., 2019, MNRAS, 487, 1380

Gentile G., Salucci P., Klein U., Vergani D., Kalberla P., 2004, MNRAS, 351, 903

Gnedin O. Y., Kravtsov A. V., Klypin A. A., Nagai D., 2004, ApJ, 616, 16

Goerdt T., Moore B., Read J. I., Stadel J., 2010, ApJ, 725, 1707

Governato F. et al., 2010, Nature, 463, 203

Governato F. et al., 2012, MNRAS, 422, 1231

Graus A. S. et al., 2019, MNRAS, 490, 1186

Griffen B. F., Ji A. P., Dooley G. A., Gómez F. A., Vogelsberger M., O'Shea B. W., Frebel A., 2016, ApJ, 818, 10

Hahn O., Abel T., 2011, MNRAS, 415, 2101

Hopkins P. F., 2015, MNRAS, 450, 53
Hopkins P. F., Kereš D., Oñorbe J., Faucher-Giguère C.-A., Quataert E., Murray N., Bullock J. S., 2014, MNRAS, 445, 581

Hopkins P. F. et al., 2018, MNRAS, 480, 800

Hunter J. D., 2007, Comput. Sci. Eng., 9, 90

Kamada A., Kaplinghat M., Pace A. B., Yu H.-B., 2017, Phys. Rev. Lett., 119,111102

Kaplinghat M., Ren T., Yu H.-B., 2020, JCAP, 027

Katz H., Lelli F., McGaugh S. S., Di Cintio A., Brook C. B., Schombert J. M., 2017, MNRAS, 466, 1648

Klypin A., Yepes G., Gottlöber S., Prada F., Heß S., 2016, MNRAS, 457, 4340

Kroupa P., 2001, MNRAS, 322, 231

Lazar A., Bullock J. S., 2020, MNRAS, 493, 5825

Leitherer C. et al., 1999, ApJS, 123, 3

Lelli F., McGaugh S. S., Schombert J. M., 2016, AJ, 152, 157

Li P., Lelli F., McGaugh S., Schombert J., 2020, ApJS, 247, 31

Ludlow A. D., Navarro J. F., Springel V., Vogelsberger M., Wang J., White S. D. M., Jenkins A., Frenk C. S., 2010, MNRAS, 406, 137

Ludlow A. D., Bose S., Angulo R. E., Wang L., Hellwing W. A., Navarro J. F., Cole S., Frenk C. S., 2016, MNRAS, 460, 1214

Macciò A. V., Crespi S., Blank M., Kang X., 2020, MNRAS, 495, L46

Madau P., Shen S., Governato F., 2014, ApJ, 789, L17

Mashchenko S., Couchman H. M. P., Wadsley J., 2006, Nature, 442, 539

Merritt D., Graham A. W., Moore B., Diemand J., Terzić B., 2006, AJ, 132, 2685

Moore B., 1994, Nature, 370, 629

Munshi F. et al., 2013, ApJ, 766, 56

Navarro J. F., Eke V. R., Frenk C. S., 1996, MNRAS, 283, L72

Navarro J. F., Frenk C. S., White S. D. M., 1997, ApJ, 490, 493

Navarro J. F. et al., 2004, MNRAS, 349, 1039

Navarro J. F. et al., 2010, MNRAS, 402, 21

Nesti F., Salucci P., 2013, JCAP, 2013, 016

Oh S.-H., Brook C., Governato F., Brinks E., Mayer L., de Blok W. J. G., Brooks A., Walter F., 2011, AJ, 142, 24

Oh S.-H. et al., 2015, AJ, 149, 180

Oliphant T. E., 2007, Comput. Sci. Eng., 9, 10

Oñorbe J., Garrison-Kimmel S., Maller A. H., Bullock J. S., Rocha M., Hahn O., 2014, MNRAS, 437, 1894

Oñorbe J., Boylan-Kolchin M., Bullock J. S., Hopkins P. F., Kereš D., FaucherGiguère C.-A., Quataert E., Murray N., 2015, MNRAS, 454, 2092

Papastergis E., Giovanelli R., Haynes M. P., Shankar F., 2015, A\&A, 574, A113

Peñarrubia J., Pontzen A., Walker M. G., Koposov S. E., 2012, ApJ, 759, L42

Planck Collaboration XIII, 2016, A\&A, 594, A13

Pontzen A., Governato F., 2012, MNRAS, 421, 3464

Portail M., Gerhard O., Wegg C., Ness M., 2017, MNRAS, 465, 1621

Power C., Navarro J. F., Jenkins A., Frenk C. S., White S. D. M., Springel V., Stadel J., Quinn T., 2003, MNRAS, 338, 14

Prada F., Klypin A. A., Simonneau E., Betancort-Rijo J., Patiri S., Gottlöber S., Sanchez-Conde M. A., 2006, ApJ, 645, 1001

Price D. J., Monaghan J. J., 2007, MNRAS, 374, 1347

Read J. I., Gilmore G., 2005, MNRAS, 356, 107

Read J. I., Agertz O., Collins M. L. M., 2016, MNRAS, 459, 2573

Relatores N. C. et al., 2019, ApJ, 887, 94

Ren T., Kwa A., Kaplinghat M., Yu H.-B., 2019, Phys. Rev. X, 9, 031020

Robles V. H., Bullock J. S., Boylan-Kolchin M., 2019, MNRAS, 483, 289

Rocha M., Peter A. H. G., Bullock J. S., Kaplinghat M., Garrison-Kimmel S., Oñorbe J., Moustakas L. A., 2013, MNRAS, 430, 81

Romano-Díaz E., Shlosman I., Hoffman Y., Heller C., 2008, ApJ, 685, L105

Salucci P., Burkert A., 2000, ApJ, 537, L9

Samuel J. et al., 2020, MNRAS, 491, 1471

Spekkens K., Giovanelli R., Haynes M. P., 2005, AJ, 129, 2119

Springel V., 2005, MNRAS, 364, 1105

Srisawat C. et al., 2013, MNRAS, 436, 150

Stinson G. S., Bailin J., Couchman H., Wadsley J., Shen S., Nickerson S., Brook C., Quinn T., 2010, MNRAS, 408, 812

Stinson G. S. et al., 2012, MNRAS, 425, 1270 
Swaters R. A., Madore B. F., van den Bosch F. C., Balcells M., 2003, ApJ, 583,732

Teyssier R., Pontzen A., Dubois Y., Read J. I., 2013, MNRAS, 429, 3068

Tollerud E. J., Boylan-Kolchin M., Bullock J. S., 2014, MNRAS, 440, 3511

Tollet E. et al., 2016, MNRAS, 456, 3542

Tonini C., Lapi A., Salucci P., 2006, ApJ, 649, 591

van der Walt S., Colbert S. C., Varoquaux G., 2011, Comput. Sci. Eng., 13, 22

Walter F., Brinks E., de Blok W. J. G., Bigiel F., Kennicutt, Robert C. J., Thornley M. D., Leroy A., 2008, AJ, 136, 2563

Wang J., Bose S., Frenk C. S., Gao L., Jenkins A., Springel V., White S. D M., 2019, preprint (arXiv:1911.09720)

Wang L., Dutton A. A., Stinson G. S., Macciò A. V., Penzo C., Kang X., Keller B. W., Wadsley J., 2015, MNRAS, 454, 83

Wetzel A. R., Hopkins P. F., Kim J.-h., Faucher-Giguère C.-A., Kereš D., Quataert E., 2016, ApJ, 827, L23

Wheeler C. et al., 2019, MNRAS, 490, 4447

Zhao H., 1996, MNRAS, 278, 488

\section{APPENDIX A: STELLAR MASS PARAMETRIZATION OF THE CORE-EINASTO}

The analysis presented in Section 4 focused on properties recovered by the core-Einasto profile and then characterizing these trends with the $M_{\star} / M_{\text {halo }}$ of the simulated FIRE-2 haloes. Here, we perform our analysis now on the stellar mass of the galaxies, $M_{\star}$, as this can provide deeper insight to observations of real galaxies comparable to the galaxies analysed in this article.

The left plot in Fig. A1 depicts the relation of $\tilde{r}_{\mathrm{s}}$ to $r_{-2}$ of the galaxies' dark matter profile as a function of $M_{\star}$. We find quite a bit of difference between this implied relationship and the relationship seen previously in Fig. 8. Primarily, the values of $\tilde{r}_{\mathrm{s}} / r_{-2}$ are more spread out for the ranges of $M_{\star}$ considered here. This is better seen with fitting the data with equation (13). Best-fitting results are given in Table 2 and are shown as the blue curve in the left plot. The right

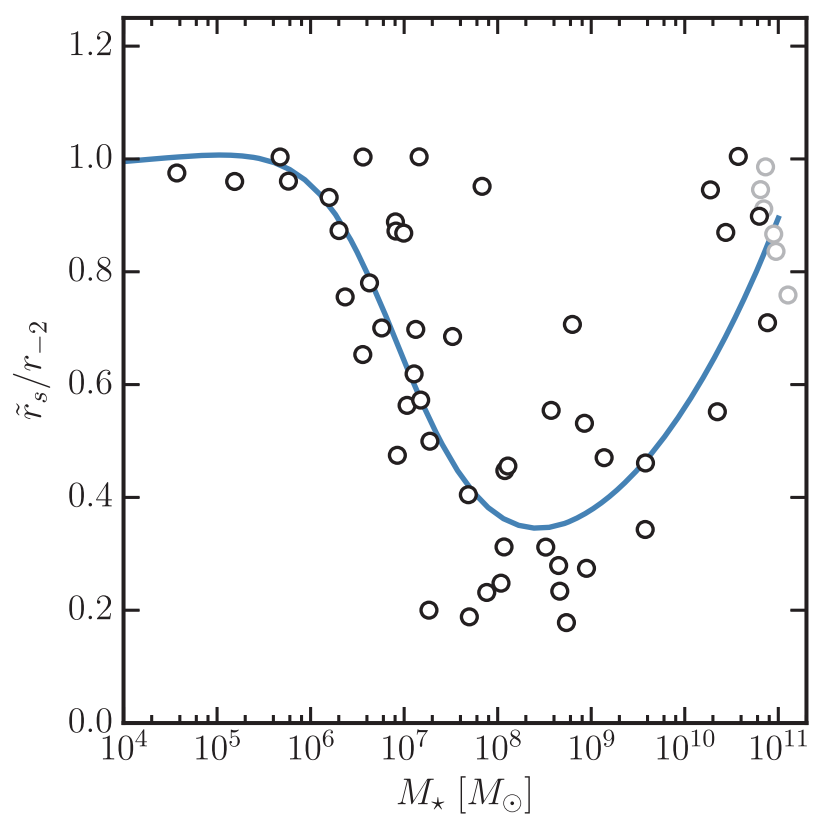

plot of Fig. A1 shows the ratio between the concentrations of the haloes for the galaxies and the DMO analogues. We consider the same definition of the concentration discussed previously in Fig. 9. The depletion in concentration spans from $M_{\star} \simeq 10^{6}-10^{9} \mathrm{M}_{\odot}$, the most prominent being at $M_{\star} \simeq 10^{7-8} \mathrm{M}_{\odot}$. The points are fitted with equation (14) with the best-fitting results, given in Table 1, are shown as the blue curve in the right plot.

\section{APPENDIX B: ANALYTICAL PROPERTIES OF CORE-EINASTO HALOES}

Here, we derive formulae in the form concerning the spatial properties of dark matter haloes described by equation (8). In the limit of $r_{\mathrm{c}} \rightarrow 0$, profiles should transform back to a cusped form, i.e. $\rho_{\text {cEin }}$ $\rightarrow \rho_{\text {Ein }}$.

\section{B1 Cumulative mass distribution}

For a spherical averaged volume, the cumulative mass is

$M(<r)=4 \pi \tilde{\rho}_{\mathrm{s}} \int_{0}^{r} \mathrm{~d} r^{\prime} r^{\prime 2} \exp \left\{-\frac{2}{\alpha_{\epsilon}}\left[\left(\frac{r^{\prime}+r_{\mathrm{c}}}{\tilde{r}_{\mathrm{s}}}\right)^{\alpha_{\epsilon}}-1\right]\right\}$.

Let us set $s=2\left(r+r_{\mathrm{c}}\right)^{\alpha_{\epsilon}} / \alpha_{\epsilon} \tilde{r}_{\mathrm{s}}^{\alpha_{\epsilon}}$, such that algebraically massaging gives us $r=s^{1 / \alpha_{\epsilon}}\left(\alpha_{\epsilon} / 2\right)^{1 / \alpha_{\epsilon}} \tilde{r}_{\mathrm{s}}-r_{\mathrm{c}}$. When substituting this into the cumulative mass expression, we have the expanded form of

$$
\begin{aligned}
M(<r)= & \frac{4 \pi \tilde{\rho}_{\mathrm{s}} e^{2 / \alpha_{\epsilon}}}{\alpha_{\epsilon}}\left\{\tilde{r}_{\mathrm{s}}^{3}\left(\frac{\alpha_{\epsilon}}{2}\right)^{3 / \alpha_{\epsilon}} \int_{s(0)}^{s(r)} \mathrm{d} s s^{3 / \alpha_{\epsilon}-1} e^{-s}\right. \\
& +r_{\mathrm{c}}^{2} \tilde{r}_{\mathrm{s}}\left(\frac{\alpha_{\epsilon}}{2}\right)^{1 / \alpha_{\epsilon}} \int_{s(0)}^{s(r)} \mathrm{d} s s^{1 / \alpha_{\epsilon}-1} e^{-s} \\
& \left.-2 r_{c} \tilde{r}_{\mathrm{s}}^{2}\left(\frac{\alpha_{\epsilon}}{2}\right)^{2 / \alpha_{\epsilon}} \int_{s(0)}^{s(r)} \mathrm{d} s s^{2 / \alpha_{\epsilon}-1} e^{-s}\right\}
\end{aligned}
$$

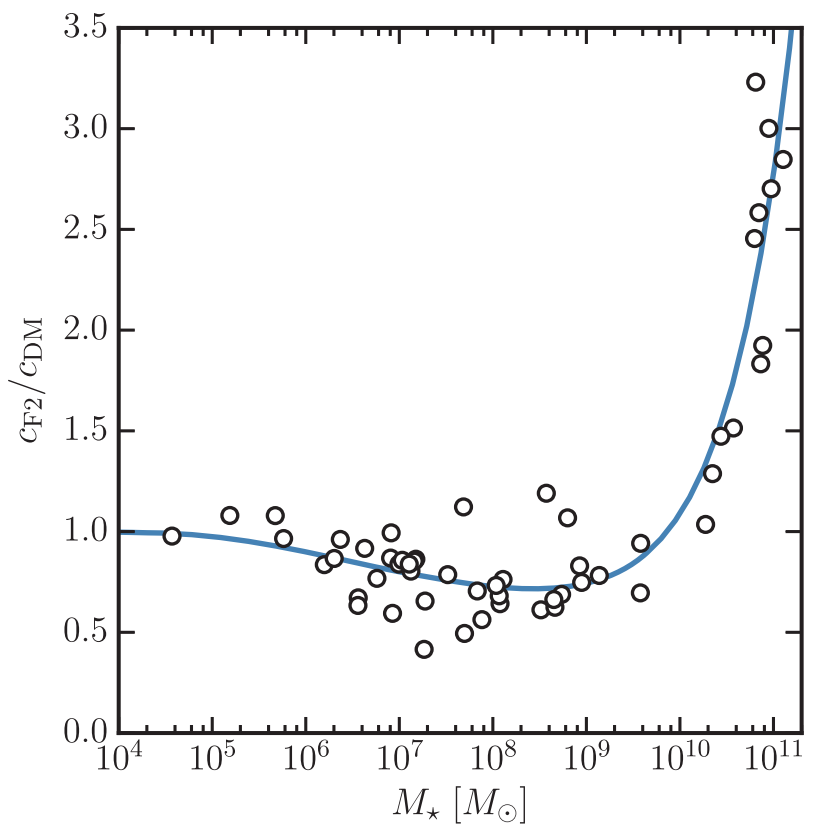

Figure A1. Halo concentration as a function of $M_{\star}$. Left: The ratio of the scale parameter $\tilde{r}_{\mathrm{s}}$ to $r_{-2}$ of the galaxy dark matter profile shows similar trends seen in earlier Fig. 9. The largest difference again correlates with the largest cores at $M_{\star}=10^{8-9} \mathrm{M}_{\odot}$. The solid blue curve depicts the fit of the points using the best-fitting parameters given in Table 2 when using equation (13) for $x=M_{\star}$. Right: The comparison between concentrations as a function of stellar mass. The reduction of concentration is apparent for a large span of $M_{\star}$. The solid blue curve depicts the fit of points using best-fitting parameters from Table 3 when using equation (14) for $x=M_{\star}$. 
We can define the integral parametrization as

$\tilde{\gamma}_{\beta}\left[x_{1}, x_{2}\right]:=\left(\frac{\alpha_{\epsilon} \tilde{r}_{\mathrm{s}}^{\alpha_{\epsilon}}}{2}\right)^{\beta} \gamma_{\beta}\left[x_{1}, x_{2}\right]$,

which is a characterization variant of the lower incomplete gamma function:

$\gamma_{\beta}\left[x_{1}, x_{2}\right]=\int_{x_{1}}^{x_{2}} \mathrm{~d} s s^{\beta-1} e^{-s}$.

This allows us to write the expression for the integrated mass in a more compact form

$$
\begin{aligned}
M(<r)= & \frac{4 \pi \tilde{\rho}_{\mathrm{s}} e^{2 / \alpha_{\epsilon}}}{\alpha_{\epsilon}}\left\{\tilde{\gamma}_{3 / \alpha_{\epsilon}}[s(0), s(r)]+r_{\mathrm{c}}^{2} \tilde{\gamma}_{1 / \alpha_{\epsilon}}[s(0), s(r)]\right. \\
& \left.-2 r_{\mathrm{c}} \tilde{\gamma}_{2 / \alpha_{\epsilon}}[s(0), s(r)]\right\} .
\end{aligned}
$$

In the limit of $r_{\mathrm{c}} \rightarrow 0$, we return back to the analytic form of the cumulative mass for the Einasto profile

$$
\begin{aligned}
M_{\operatorname{Ein}}(<r) & =\lim _{r_{\mathrm{c}} \rightarrow 0} M_{\mathrm{cEin}}(<r) \\
& =\frac{4 \pi \tilde{\rho}_{\mathrm{s}} e^{2 / \alpha_{\epsilon}} \tilde{r}_{\mathrm{s}}^{3}}{\alpha_{\epsilon}}\left(\frac{\alpha_{\epsilon}}{2}\right)^{3 / \alpha_{\epsilon}} \gamma_{3 / \alpha_{\epsilon}}[0, s(r)],
\end{aligned}
$$

where we then retrieve the lower incomplete gamma function in this limit

$\gamma_{\beta}[0, x]=\int_{0}^{x} \mathrm{~d} s s^{\beta-1} e^{-s}$.

\section{B2 Gravitational potential}

The gravitational potential of a spherically symmetric mass distribution, $\rho(r)$, can be found through the expression (Binney \& Tremaine 2008),

$\Psi(r)=4 \pi G\left[\int_{0}^{r} \mathrm{~d} r^{\prime} r^{\prime 2} \rho\left(r^{\prime}\right)+\int_{r}^{\infty} \mathrm{d} r^{\prime} r^{\prime} \rho\left(r^{\prime}\right)\right]$.

It follows for the cored-Einasto,

$$
\begin{aligned}
& \Psi(r)=\frac{4 \pi G \tilde{\rho}_{\mathrm{s}} e^{2 / \alpha_{\epsilon}}}{\alpha_{\epsilon}}\left\{\frac { 1 } { r } \left(\tilde{\gamma}_{3 / \alpha_{\epsilon}}[s(0), s(r)]\right.\right. \\
& \left.+r_{\mathrm{c}}^{2} \tilde{\gamma}_{1 / \alpha_{\epsilon}}[s(0), s(r)]-2 r_{\mathrm{c}} \tilde{\gamma}_{2 / \alpha_{\epsilon}}[s(0), s(r)]\right) \\
& \left.+\tilde{\Gamma}_{2 / \alpha_{\epsilon}}[s(r)]-r_{\mathrm{c}} \tilde{\Gamma}_{1 / \alpha_{\epsilon}}[s(r)]\right\},
\end{aligned}
$$

where we have defined

$$
\tilde{\Gamma}_{\beta}[s(r)]=\left(\frac{\alpha_{\epsilon} \tilde{r}_{s}^{\alpha_{\epsilon}}}{2}\right)^{\beta} \Gamma_{\beta}[s(r)],
$$

such that

$\Gamma_{\beta}[x]=\int_{x}^{\infty} \mathrm{d} s s^{\beta-1} s^{-s}$

is the upper incomplete Gamma function.

\section{B3 Energy of induced core formation}

The transformation from a cusp inner region to a core is presumed to be from highly energetic stellar feedback. After the dark matter cusp is removed we would infer that the halo settles in a new equilibrium state. Dark matter in dynamical equilibrium will then satisfy the virial theorem, i.e. $E=\mathcal{W} / 2$. Here, $\mathcal{W}$ is the magnitude of the gravitational potential energy associated with the mass distribution:

$\mathcal{W}=-\int_{0}^{r_{\mathrm{vir}}} \mathrm{d} r^{\prime} \frac{G M\left(<r^{\prime}\right)}{r^{\prime}} 4 \pi r^{\prime 2} \rho\left(r^{\prime}\right)$.

For the core-Einasto, the gravitational energy is

$$
\begin{aligned}
\mathcal{W}_{\mathrm{cEin}}= & -\left(\frac{16 \pi^{2} G^{2} \tilde{\rho}_{\mathrm{s}}^{2} e^{4 / \alpha_{\epsilon}}}{\alpha_{\epsilon}}\right) \int_{0}^{r_{\mathrm{vir}}} \mathrm{d} r^{\prime} e^{-s\left(r^{\prime}\right)} \times \\
& \left\{\tilde{\gamma}_{3 / \alpha_{\epsilon}}\left[s(0), s\left(r^{\prime}\right)\right]+r_{\mathrm{c}}^{2} \tilde{\gamma}_{1 / \alpha_{\epsilon}}\left[s(0), s\left(r^{\prime}\right)\right]\right. \\
& \left.-2 r_{\mathrm{c}} \tilde{\gamma}_{2 / \alpha_{\epsilon}}\left[s(0), s\left(r^{\prime}\right)\right]\right\},
\end{aligned}
$$

while for the cusp nature, the Einasto profile has

$$
\begin{aligned}
\mathcal{W}_{\text {Ein }}= & -\left(\frac{16 \pi^{2} G^{2} \rho_{-2}^{2} e^{4 / \alpha_{\epsilon}}}{\alpha_{\epsilon}}\right) \int_{0}^{r_{\text {vir }}} \mathrm{d} r^{\prime} \exp \left[\frac{2}{\alpha_{\epsilon}}\left(\frac{r^{\prime}}{r_{-2}}\right)^{\alpha_{\epsilon}}\right] \\
& \times\left(\frac{2 r_{-2}^{2}}{\alpha_{\epsilon}}\right)^{3 / \alpha_{\epsilon}} \gamma_{3 / \alpha_{\epsilon}}\left[0, \frac{2}{\alpha_{\epsilon}}\left(\frac{r^{\prime}}{r_{-2}}\right)^{\alpha_{\epsilon}}\right] .
\end{aligned}
$$

Analytically, we can then quantify a conservative limit for the lower bound of energy needed to transform the inner density via the virial theorem, i.e.

$\Delta E=\frac{\Delta \mathcal{W}}{2}=\frac{\mathcal{W}_{\text {cEin }}-\mathcal{W}_{\text {Ein }}}{2}$

\section{APPENDIX C: A PROFILE FOR BARYONIC CONTRACTED HALOES}

A major focus of this work is that equation (8), $\rho_{\text {cEin }}$, characterizes dark matter profiles with dark matter cores. While a majority of the dwarf galaxies in our sample are well described by $\rho_{\text {cEin }}$, a majority of our MW-mass haloes (not including $\mathrm{m} 12 \mathrm{w}, \mathrm{m} 12 \mathrm{z}$, Louise, and Thelma) are not well fitted by this profile given the inaccurate results of $r_{\mathrm{c}}$. This seems to happen for MW-mass haloes that have small cores garnished with baryonic contraction to their dark matter distribution in the innermost regions. This motivates us to come up with a profile that accommodates both of these features in galaxies that are this massive.

We would guess that the amplitude of a baryonic-contracted halo has the density amplitude be radially dependent:

$\tilde{\rho}_{\mathrm{s}, \mathrm{BC}}(r)=\tilde{\rho}_{\mathrm{s}}\left[1+X \cdot \tanh \left(\frac{r_{\mathrm{c}}}{r}\right)\right]$,

which contributes to the profile at small radii. Here, $X$ is some free variable in the fit that is added to compensate for unusual amplitudes in several of the MW-mass haloes. This is written in a way such that at $r_{\mathrm{c}}=0$, we only have have $\tilde{\rho}_{\mathrm{s}, \mathrm{BC}}=\tilde{\rho}_{\mathrm{s}}=\rho_{-2}$, and at $r=0$, we have $\tilde{\rho}_{s, \mathrm{BC}}=\tilde{\rho}_{s}(1+X)$. It would then

$\rho_{\text {cEin, BC }}(r)=\tilde{\rho}_{\mathrm{s}, \mathrm{BC}}(r) \times \exp \left\{-\frac{2}{\alpha_{\epsilon}}\left[\left(\frac{r+r_{\mathrm{c}}}{\tilde{r}_{\mathrm{s}}}\right)^{\alpha_{\epsilon}}-1\right]\right\}$.

Additionally, this allows us to parametrize the central core density similar to equation (9):

$\rho_{0, \mathrm{BC}}:=\rho_{\text {cEin, BC }}(0)=[1+X] \rho_{0}$.

Fig. $\mathrm{C} 1$ plots the results for fitting $\rho_{\text {cEin, BC }}$ (the dashed pink curve) to several of the FIRE-2 MW-mass haloes (the solid black curve). Also plotted is the DMO analogue as the grey curve. The value of $r_{\mathrm{c}}$ predicted by $\rho_{\text {cEin, } \mathrm{BC}}$ is highlighted in the same colour and pointed to with its $r_{\text {vir }}$ normalization. We list our values for these fits in Table $\mathrm{C} 1$. 


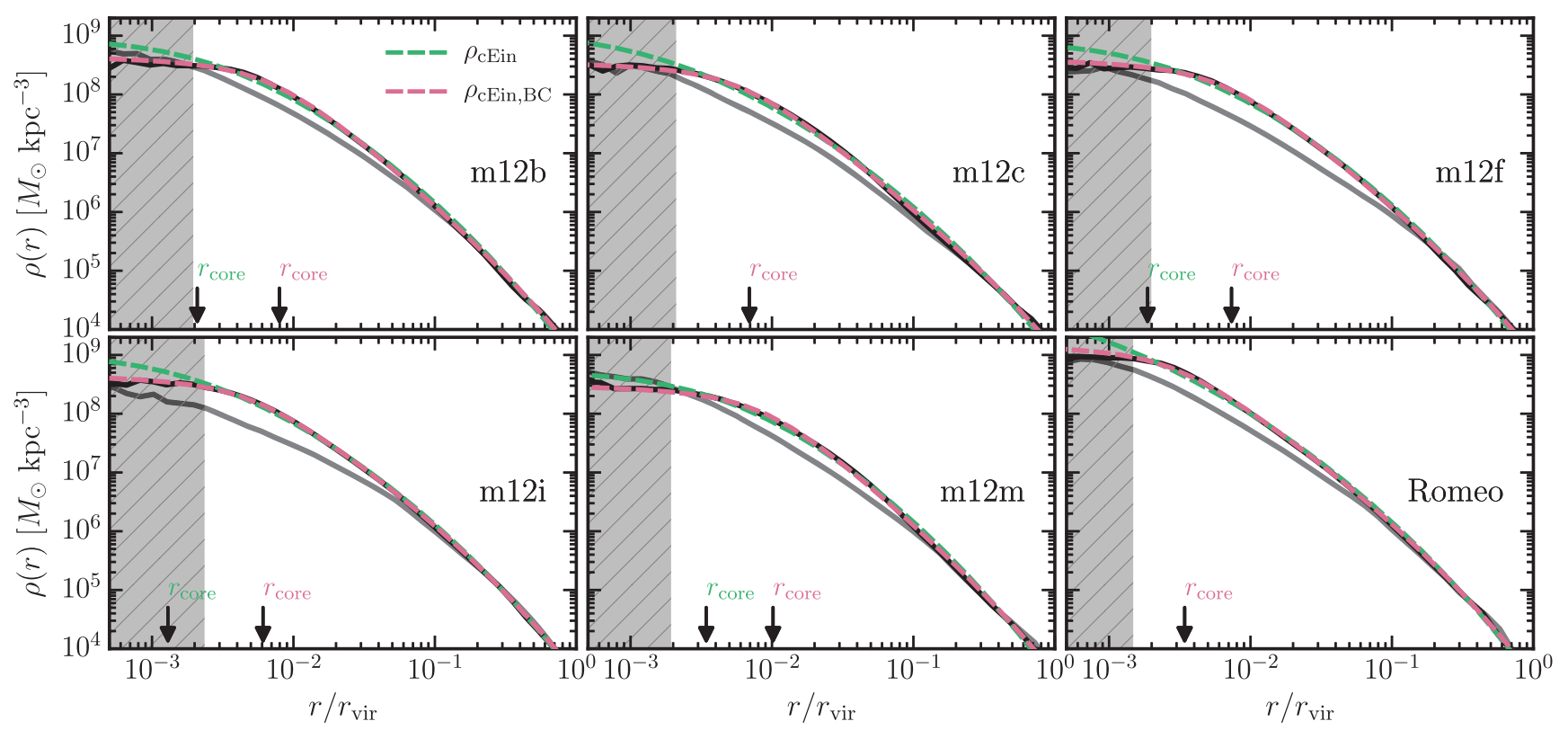

Figure C1. Refined profiles for cored MW-mass haloes. As in Fig. 4, galaxies are shown as the solid black curves while their DMO analogues are the solid grey curves. The original $\rho_{\text {cEin }}$ fits are plotted as the green-dashed curves, while $\rho_{\text {cEin, BC }}$ fits are plotted as the pink-dashed curves. The location of the resulting core radius of each galaxy from both fits is indicate by an arrow with the corresponding colour. We see that a radially dependent density component in $\rho_{\text {cEin, }} \mathrm{BC}$ greatly improves the fits while also accurately predicting the core radius.

Table C1. Best-fitting parameters for Milky Way-mass haloes.

\begin{tabular}{lccccc}
\hline $\begin{array}{l}\text { Halo } \\
\text { name }\end{array}$ & $\begin{array}{c}\tilde{\rho}_{\mathrm{s}} \\
\left(\mathrm{M}_{\odot} \mathrm{kpc}^{-3}\right)\end{array}$ & $\begin{array}{c}\tilde{r}_{\mathrm{s}} \\
(\mathrm{kpc})\end{array}$ & $X$ & $\begin{array}{c}r_{\mathrm{c}} \\
(\mathrm{kpc})\end{array}$ & $Q_{\text {min }}$ \\
\hline $\mathrm{m} 12 \mathrm{~b}$ & $1.1 \times 10^{6}$ & 21.2 & 5.44 & $\checkmark_{1.77}$ & 0.0236 \\
$\mathrm{~m} 12 \mathrm{c}$ & $4.4 \times 10^{5}$ & 31.5 & 5.50 & $\checkmark_{1.52}$ & 0.0349 \\
$\mathrm{~m} 12 \mathrm{f}$ & $1.3 \times 10^{6}$ & 21.3 & 3.73 & $\checkmark_{1.73}$ & 0.0230 \\
$\mathrm{~m} 12 \mathrm{i}$ & $2.0 \times 10^{6}$ & 16.5 & 2.37 & $\checkmark_{1.28}$ & 0.0085 \\
$\mathrm{~m} 12 \mathrm{~m}$ & $1.0 \times 10^{6}$ & 22.1 & 5.50 & $\checkmark_{2.31}$ & 0.0306 \\
Romeo & $2.8 \times 10^{6}$ & 15.1 & 3.54 & $\checkmark_{0.76}$ & 0.0168 \\
Juliet & $1.6 \times 10^{6}$ & 17.2 & 3.93 & $\checkmark_{0.70}$ & 0.0187 \\
\hline
\end{tabular}

Note. Use equation (C2) with $\alpha_{\epsilon}=0.16$.

We can see that for MW-mass haloes with both baryonic contraction and a physical core, $\rho_{\text {cEin, } \mathrm{BC}}$, while not particularly succinct, is the most ideal function we can use to probe $r_{\mathrm{c}}$. However, the exact behaviour and physical interpretation of $\tilde{r}_{\mathrm{s}}$ is left, now, somewhat ambiguous compared to how it was expected to behave previously in Section 4. The same MW-mass haloes that have had their core radii previously predicted with $\rho_{\text {cEin }}$ are also plotted in Fig. $\mathrm{C} 1$ as the green-dashed curve. The predicted core radius from this profile is pointed to and highlighted in green. From direct comparison between the analytical fits, we see significant improvements. We have included $r_{\mathrm{c}}$ values here in the main text as the cyan points in Figs 7 and 8 .

\section{APPENDIX D: COMPARISON WITH OTHER DARK MATTER PROFILES}

Here, we compare several other dark matter profiles in the literature and compare their fits to our FIRE-2 simulation sample:

(i) P12 (Peñarrubia et al. 2012): One commonly adopted dark matter profile that is an extension of the two-parameter NFW profile that accommodates a physical core radius:

$\rho_{\mathrm{P} 12}(r)=\frac{\rho_{0} r_{0}^{3}}{\left(r_{\mathrm{c}}+r\right)\left(r_{0}+r\right)^{2}}$,

where $\rho_{0}$ is the characteristic scale density and $r_{0}$ is some scale radius. The form of equation (D1) transforms back to an NFW profile in the limit of $r_{\mathrm{c}} \rightarrow 0$. The form of equation (D1) is a three-parameter profile with free variables $\rho_{0}, r_{0}$, and $r_{\mathrm{c}}$. Equation (D1) is fitted with the FIRE-2 haloes by utilizing the fitting routine discussed in Section 4 and best-fitting parameters are obtained by minimizing the figure of merit, equation (11).

(ii) $\alpha \beta \gamma$ (Zhao 1996): A generic five parameter profile dubbed the ' $\alpha \beta \gamma$-profile':

$\rho_{\alpha \beta \gamma}(r)=\frac{\rho_{\mathrm{s}}}{\left(r / r_{\mathrm{s}}\right)^{\gamma_{s}}\left[1+\left(r / r_{\mathrm{s}}\right)^{\alpha_{\mathrm{s}}}\right]^{\left(\beta_{s}-\gamma_{\mathrm{s}}\right) / \alpha_{\mathrm{s}}}}$,

where $r_{\mathrm{s}}$ is the scale radius and $\rho_{\mathrm{s}}$ is the scale density. The inner and outer regions are parametrized, respectively, by the logarithmic slopes, $-\gamma_{\mathrm{s}}$ and $-\beta_{\mathrm{s}}$, while $\alpha_{\mathrm{s}}$ controls the rate of transition from the inner and outer region. The form of equation (D2) has five freeparameters $\rho_{\mathrm{s}}, r_{\mathrm{s}}, \alpha_{\mathrm{s}}, \beta_{\mathrm{s}}$, and $\gamma_{\mathrm{s}}$. equation (D2) is fitted with the FIRE-2 haloes by utilizing the routine discussed in Section 4 and best-fitting parameters are obtained by minimizing the figure of merit, equation (11).

(iii) DC14 (Di Cintio et al. 2014b): The DC14 model takes the generalized form of equation (D2) and imposes dependence of the slope parameters as a function of $M_{\star} / M_{\text {halo }}$ :

$$
\begin{aligned}
& \alpha_{\mathrm{s}}(X)=2.94-\log _{10}\left[\left(10^{X+2.33}\right)^{-1.08}+\left(10^{X+2.33}\right)^{2.29}\right], \\
& \beta_{\mathrm{s}}(X)=4.23+1.34 X+0.26 X^{2} \\
& \gamma_{\mathrm{s}}(X)=-0.06+\log _{10}\left[\left(10^{X+2.56}\right)^{-0.68}+\left(10^{X+2.56}\right)\right]
\end{aligned}
$$



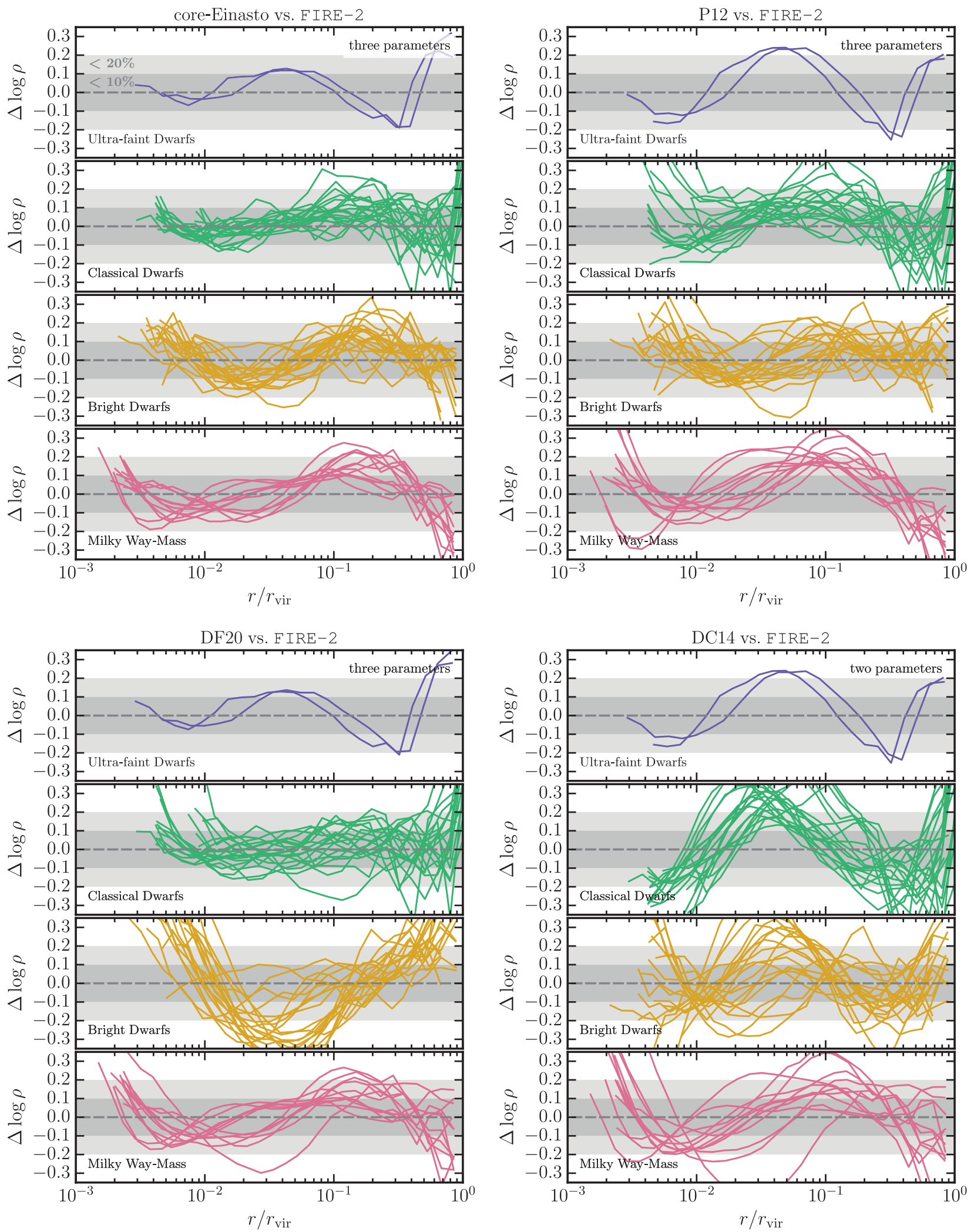

Figure D1. Fit residuals, as in Fig. 5: residuals for core-Einasto (top left), P12 (top right), DF20 (bottom left), and DC14 (bottom right) profiles for our halo sample. The number of free parameters in each fit is indicated in the upper right of each panel. See Appendix D for the definition of P12, DF20, and DC14. 


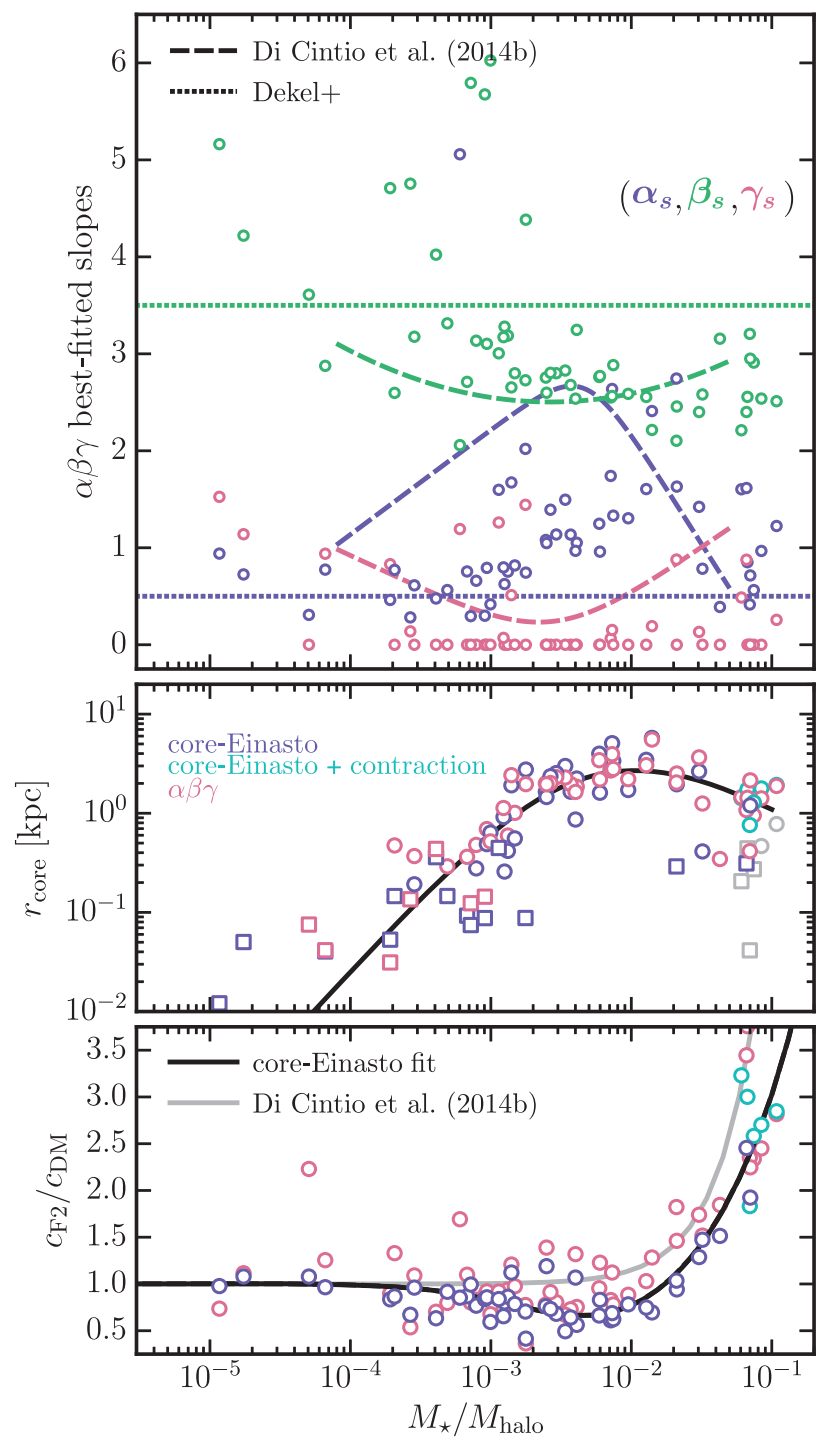

Figure D2. Properties from the $\alpha \beta \gamma$-profile fits. Top panel: The best-fitting results for the inner slope $\left(\gamma_{\mathrm{s}}\right.$; pink), the outer slope $\left(\beta_{\mathrm{s}}\right.$; green), and the transitioning slope $\left(\alpha_{s} ;\right.$ blue). Middle panel: The core radius as parametrized from the characteristic radius, $r_{-1}$, from the best-fitting $\alpha \beta \gamma$-profiles via equation (D6). We assumed that $r_{-1}$ acts as a probe of $r_{\mathrm{c}}$ and find agreeable results from $r_{\mathrm{c}}$ derived by fitting equation (8) in the main text. Bottom panel: The ratio between the concentration parameter for the FIRE-2 haloes and their DMO analogues using equation (D7). Shown are the core-Einasto (blue) and core-Einasto with contraction (cyan) values presented previously in Fig. 10. Both the FIRE-2 haloes and DMO analogues are fitted with the $\alpha \beta \gamma$-profile to obtain the concentration parameters shown in pink. The concentration ratio is mostly in agreement with slight differences at the edge of the bright dwarf regime $\left(M_{\star} / M_{\text {halo }} \approx 10^{-2}\right)$.

where $X:=\log _{10}\left(M_{\star} / M_{\text {halo }}\right)$ and is valid in the range of $-4.1<$ $X<-1.3$. Outside this mass range resorts to a NFW profile, i.e. $\left(\alpha_{\mathrm{s}}, \beta_{\mathrm{s}}, \gamma_{\mathrm{s}}\right)=(1,3,1)$. This now leaves equation (D2) with two free-parameters: $r_{\mathrm{s}}$ and $\rho_{\mathrm{s}}$. The DC14 profile is fitted with the FIRE2 haloes by utilizing the routine discussed in Section 4 and bestfitting parameters are obtained by minimizing the figure of merit, equation (11).

(iv) DF20 (Dekel et al. 2017; Freundlich et al. 2020): The DF20 model (or the "Dekel + "' profile) takes the generic double powerlaw density profile, namely equation (D2), and has fixed slopes $\alpha_{\mathrm{s}}=$
0.5 and $\beta_{\mathrm{s}}=3.5$. This reduces the analytical profile to be fitted based on three free-parameters: $\rho_{\mathrm{s}}, r_{\mathrm{s}}$, and $\gamma_{\mathrm{s}}$. The form of DF20 is fitted with the FIRE- 2 haloes by utilizing the routine discussed in Section 4 and best-fitting parameters are obtained by minimizing the figure of merit, equation (11).

We also attempted a similar analysis using the core profile from Read et al. (2016) with their four free-parameters and found the resulting fits incompatible with our simulated profiles.

\section{D1 Resulting profile residuals}

Fig. D1 compares the residuals of the FIRE-2 dark matter haloes when fitted with the core-Einasto (top left, same fits as presented in the main text), P12 (top right), DF20 (bottom left), and the $\alpha \beta \gamma$ model with DC14 parametrization (bottom right). The interesting comparison to be made is between core-Einasto and P12 since both profiles have three parameters to be determined, with one being the core radius of the dark matter halo. We see the the core-Einasto does better at fitting the FIRE-2 dark matter haloes than the P12 shape. Although P12 does not do as well as fitting to our FIRE-2 haloes, the form P12 has the advantage of being more analytically friendly when quantifying characteristics of the dark matter halo. For the DF20 model, we find that it is comparable with the core-Einasto fits for the classifications of the ultrafaints, classical dwarfs, and most MW haloes. However, DF20 fails to capture the shape of our bright galaxies, i.e. the haloes with the largest feedback-induced cores.

Results for the two-parameter DC14 model are shown to be poor fits with the FIRE-2 haloes for all of our mass range. This likely has to to do with differences in the dark matter distribution found at fixed stellar mass fractions compared to the simulations explored in DC14.

\section{D2 Extended analysis with $\alpha \beta \gamma$}

Unsurprisingly, the five-parameter $\alpha \beta \gamma$ model provides a superior fit for a majority of our simulated haloes compared to our threeparameter core-Einasto profile. Specifically, we find that this profile can do better than 10 per cent for almost all of our galaxies. While the resulting $\alpha \beta \gamma$-profile fits model the dark matter distribution well, the physical interpretation of the resulting best-fitting parameters is less clear. A majority of the fits favour a inner-slope of $\gamma_{\mathrm{s}}=0$ for several cusped profiles, which at times imposes too large of a scale radius to be determined (m10d, m10i, and m10j to name a few). Regardless of the physical interpretation of the resulting parameter fits, we find excellent accuracy modelling our haloes, which still enables us to extract characteristics of the best-fitting profiles. In the next section, we perform a brief analysis on the best-fitting parameters for the $\alpha \beta \gamma$-profile.

In Fig. D2, we present the results when using the $\alpha \beta \gamma$-profile as function of the stellar mass fraction, $M_{\star} / M_{\text {halo }}$. The bets-fitting parameter fits the $\alpha \beta \gamma$ are also presented for each of our haloes in Fig. E2.

\section{D2.1 Best-fitting slopes}

The top panel shows the best-fitting results for the inner slope $\left(\gamma_{\mathrm{s}}\right.$; pink), the outer slope $\left(\beta_{s}\right.$; green), and the transitioning slope $\left(\alpha_{\mathrm{s}}\right.$; blue). Also plotted are the trend of the slopes from Di Cintio et al. (2014a) as the dashed curves and the dotted curves are having the fixed slopes, $\alpha_{\mathrm{s}}=0.5$ and $\beta_{\mathrm{s}}=3.5$, as suggest by Dekel et al. (2017) and Freundlich et al. (2020). Noticeably, the inner-slope, $\alpha_{\mathrm{s}}$, tends 


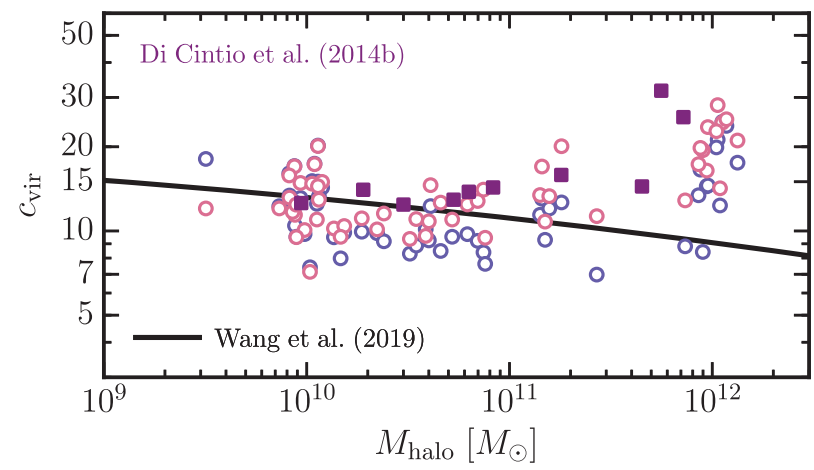

Figure D3. Concentration as a function of dark matter halo mass. Concentration implied from the core-Einasto profile are given by the blue points while the concentration shown from $\alpha \beta \gamma$-profile fits, i.e. using equation (D7), is given in pink. The solid black curve is the concentration relation from Wang et al. (2019). The purple squares are the results taken from Di Cintio et al. (2014b).

to chooses to be zero as a best-fitting parameter for a majority of our galaxies. For $\beta_{\mathrm{s}}$ and $\gamma_{\mathrm{s}}$, a sufficient amount of scatter is seen from the due to allowed large number free-parameters allowed to be fit. Although, trends as a function of $M_{\star} / M_{\text {halo }}$, can be somewhat made out.

\section{D2.2 Core radius parametrization}

Notice that in Fig. 3, the log-slope profile tends to rise at around $\operatorname{dlog} \rho / \mathrm{d} \log r \simeq-1$, which happens at the radius $r_{-1}$. To play with the idea that the physical core radius can be parametrized by the $\alpha \beta \gamma$-profile, we see how $r_{-1}$ is able to probe the core radius, i.e.

$r_{\mathrm{c}} \simeq r_{-1}=\left(\frac{1-\gamma_{\mathrm{s}}}{\beta_{\mathrm{s}}-1}\right)^{1 / \alpha_{\mathrm{s}}} r_{\mathrm{s}}$.

In the middle panel of Fig D2, we plot the previous core radius results from Fig. 7 for core-Einasto (the blue points), the baryonic contracted core-Einasto (the cyan points), and the median fit (the black curve) while also included the $\alpha \beta \gamma$-profile results using the assumed relation from equation (D6). We see that the $r_{\mathrm{c}}$ parametrization from the $\alpha \beta \gamma$-profile follows the median $r_{\mathrm{c}}$ curve from core-Einasto parametrization extremely well, implying both excellent agreement with our fitted $r_{\mathrm{c}}$ results from core-Einasto and how $r_{-1}$ characterizes the core radius.

\section{D2.3 Halo concentration}

Presented in the bottom panel of Fig D2 is the ratio between the concentration parameter of the FIRE-2 haloes and their DMO analogues. As noted in Di Cintio et al. (2014b), the radius at which the log-slope of the generic five-parameter profile is equal to -2 , $r_{-2}$, is mapped from the free-parameters via

$r_{-2}=\left(\frac{2-\gamma_{\mathrm{s}}}{\beta_{s}-2}\right)^{1 / \alpha_{\mathrm{s}}} r_{\mathrm{s}}$.

Shown are the points of the core-Einasto (blue) and core-Einasto with contraction (cyan), which are the same values depicted in Fig. 10 of the main text, while the pink points are the concentration parameters, which is still defined as $c_{\mathrm{vir}}=r_{\mathrm{vir}} / r_{-2}$, for the best-fitting $\alpha \beta \gamma$ profiles. Note that both the FIRE-2 haloes and the DMO haloes are fitted with $\alpha \beta \gamma$-profile to quantify the $r_{-2}$ values. We mostly find agreement with either methods of quantifying the halo concentration, though several of the haloes in the stellar mass fraction range of $10^{-5}-10^{-3}$ are strongly scattered. In the classical dwarf regime, we find haloes that are less concentrated like we found from the coreEinasto model. We somewhat find agreement in the bright dwarf regime, although a sufficient of scatter is present. Though recently, Freundlich et al. (2020) reports a similar result at this stellar mass fraction. The MW haloes are mostly consistent with our previous findings in the main text.

A different viewed of the concentration parameter can be made by taking the previous points and plotting as a function of $M_{\text {halo }}$ in Fig. D3. The purple squares are the haloes presented in Di Cintio et al. (2014b).

\section{APPENDIX E: SIMULATION SAMPLE}

Presented in Tables E1 and E2 are the suite of haloes simulated using FIRE-2 with their relevant parameters listed at $z=0$.

\section{E1 Global and simulation properties}

Presented in Table E1 are the global properties of the FIRE-2 galaxies at $z=0$ as well as the relevant simulation properties. Columns (15) contain global properties of the galaxies, while columns (6-10) describe the numerical resolution properties of the simulations. All simulations were ran using with $n_{\text {crit }}=10^{3} \mathrm{~cm}^{-3}$, the minimum gas density required for star formation in addition to self-shielding, Jeans instability, and self-gravity. References, given in the last column, are labelled as such - A: Fitts et al. (2017), B: Graus et al. (2019), C: Wheeler et al. (2019), D: Chan et al. (2018), E: El-Badry et al. (2018a), F: Hopkins et al. (2018), G: Garrison-Kimmel et al. (2019), H: Samuel et al. (2020), I: Wetzel et al. (2016). The individual columns in Table E1 are described as follows:

(1) $M_{\text {halo }}$ : The mass of the target halo at $z=0$ defined by Bryan $\&$ Norman (1998).

(2) $r_{\text {vir }}$ : The virial radius in physical units of the target halo.

(3) $V_{\max }$ : The maximum circular velocity curve for the dark matter component of the FIRE-2 dark matter haloes, i.e. $V_{\max }:=\max \left[V_{\text {circ }}\right]$.

(4) $M_{\star}$ : Stellar mass (within 10 per cent of $r_{\mathrm{vir}}$ ) of the central galaxy in the target halo.

(5) $r_{1 / 2}$ : The physical radius that encloses half the value of $M_{\star}$ for the central galaxy.

(6) $m_{\mathrm{b}}$ : The mass of baryon particles of the simulation.

(7) $m_{\mathrm{dm}}$ : The mass of dark matter particles of the simulation.

(8) $\epsilon_{\mathrm{dm}}$ : The dark matter force softening

(9) $r_{\text {conv }}$ : Radius of numerical convergence of the DMO analogues, set by equation (1) and the most conservative criterion as discussed in Hopkins et al. (2018) .

\section{E2 Resulting analytical profile fits}

Presented in Table E2 are the best-fitting parameters for the coreEinasto and $\alpha \beta \gamma$-profile. Columns (1-5) are the results of fitting the simulated density profiles to the core-Einastro profile with $\alpha_{\epsilon}=$ 0.16 . Columns (6-9) are the results of fitting the simulated density profiles to the $\alpha \beta \gamma$-profile. The individual columns in Table E2 are described as follows:

(1) $\tilde{\rho}_{\mathrm{s}}$ : The scale density fitted as a free parameter for the coreEinasto profile, equation (8).

(2) $\tilde{r}_{\mathrm{s}}$ : The scale radius fitted as a free parameter for the coreEinasto profile, equation (8). 
Table E1. Global parameters of the FIRE-2 haloes.

\begin{tabular}{|c|c|c|c|c|c|c|c|c|c|c|}
\hline $\begin{array}{l}\text { Halo } \\
\text { name }\end{array}$ & $\begin{array}{c}M_{\text {halo }} \\
\left(\mathrm{M}_{\odot}\right) \\
(1)\end{array}$ & $\begin{array}{c}r_{\mathrm{vir}} \\
(\mathrm{kpc}) \\
(2)\end{array}$ & $\begin{array}{c}V_{\max } \\
\left(\mathrm{km} \mathrm{s}^{-1}\right) \\
(3)\end{array}$ & $\begin{array}{c}M_{\star} \\
\left(\mathrm{M}_{\odot}\right) \\
(4)\end{array}$ & $\begin{array}{c}r_{1 / 2} \\
(\mathrm{kpc}) \\
(5)\end{array}$ & $\begin{array}{c}m_{\mathrm{b}} \\
\left(\mathrm{M}_{\odot}\right) \\
(6)\end{array}$ & $\begin{array}{c}m_{\mathrm{dm}} \\
\left(\mathrm{M}_{\odot}\right) \\
(7)\end{array}$ & $\begin{array}{c}\epsilon_{\mathrm{dm}} \\
(\mathrm{pc}) \\
(8)\end{array}$ & $\begin{array}{c}r_{\text {conv }} \\
(\mathrm{kpc}) \\
(9)\end{array}$ & Reference \\
\hline \multicolumn{11}{|c|}{ Ultrafaint dwarfs (2) } \\
\hline $\mathrm{m} 10 \mathrm{v}_{250}$ & $8.9 \times 10^{9}$ & 57.7 & 30 & $1.5 \times 10^{5}$ & 0.35 & 250 & 1300 & 29 & 0.166 & $\mathrm{C}$ \\
\hline $\mathrm{m} 10 \mathrm{v}_{250} \mathrm{~B}$ & $3.2 \times 10^{9}$ & 40.9 & 24 & $3.7 \times 10^{4}$ & 0.42 & 250 & 1300 & 29 & 0.153 & $\mathrm{C}$ \\
\hline \multicolumn{11}{|c|}{ Classical dwarfs (20) } \\
\hline $\mathrm{m} 10 \mathrm{~b}$ & $9.3 \times 10^{9}$ & 54.8 & 31 & $4.7 \times 10^{5}$ & 0.34 & 500 & 2500 & 50 & 0.218 & A \\
\hline $\mathrm{m} 10 \mathrm{c}$ & $8.8 \times 10^{9}$ & 54.1 & 31 & $5.8 \times 10^{5}$ & 0.35 & 500 & 2500 & 50 & 0.227 & A \\
\hline m10d & $8.2 \times 10^{9}$ & 50.7 & 32 & $1.6 \times 10^{6}$ & 0.53 & 500 & 2500 & 50 & 0.209 & $\mathrm{~A}$ \\
\hline $\mathrm{m} 10 \mathrm{e}$ & $9.8 \times 10^{9}$ & 53.8 & 31 & $2.0 \times 10^{6}$ & 0.62 & 500 & 2500 & 50 & 0.216 & $\mathrm{~A}$ \\
\hline m10f & $8.7 \times 10^{9}$ & 51.5 & 35 & $4.7 \times 10^{6}$ & 0.75 & 500 & 2500 & 50 & 0.202 & A \\
\hline $\mathrm{m} 10 \mathrm{~g}$ & $7.3 \times 10^{9}$ & 48.6 & 32 & $5.7 \times 10^{6}$ & 0.95 & 500 & 2500 & 50 & 0.215 & A \\
\hline $\mathrm{m} 10 \mathrm{~h}$ & $1.2 \times 10^{10}$ & 57.2 & 37 & $8.1 \times 10^{6}$ & 0.83 & 500 & 2500 & 50 & 0.207 & A \\
\hline $\mathrm{m} 10 \mathrm{i}$ & $1.1 \times 10^{10}$ & 56.3 & 40 & $8.2 \times 10^{6}$ & 0.57 & 500 & 2500 & 50 & 0.195 & A \\
\hline $\mathrm{m} 10 \mathrm{j}$ & $1.1 \times 10^{10}$ & 55.4 & 37 & $9.9 \times 10^{6}$ & 0.70 & 500 & 2500 & 50 & 0.194 & A \\
\hline $\mathrm{m} 10 \mathrm{k}$ & $1.1 \times 10^{10}$ & 56.4 & 38 & $1.1 \times 10^{7}$ & 1.14 & 500 & 2500 & 50 & 0.207 & A \\
\hline m101 & $1.1 \times 10^{10}$ & 56.1 & 37 & $1.3 \times 10^{7}$ & 0.78 & 500 & 2500 & 50 & 0.202 & A \\
\hline $\mathrm{m} 10 \mathrm{~m}$ & $1.1 \times 10^{10}$ & 56.1 & 38 & $1.5 \times 10^{7}$ & 0.96 & 500 & 2500 & 50 & 0.208 & A \\
\hline $\mathrm{m} 10 \mathrm{q}_{250}$ & $8.2 \times 10^{9}$ & 56.2 & 33 & $2.3 \times 10^{6}$ & 0.81 & 250 & 1300 & 29 & 0.150 & $\mathrm{C}$ \\
\hline $\mathrm{m} 10 \mathrm{xc}_{\mathrm{A}}$ & $8.5 \times 10^{9}$ & 53.1 & 35 & $8.5 \times 10^{6}$ & 1.80 & 4000 & 20000 & 100 & 0.455 & B \\
\hline $\mathrm{m} 10 \mathrm{xd}_{\mathrm{A}}$ & $2.4 \times 10^{10}$ & 75.5 & 38 & $1.4 \times 10^{7}$ & 1.90 & 4000 & 20000 & 100 & 0.476 & B \\
\hline $\mathrm{m} 10 \mathrm{xe}_{\mathrm{A}}$ & $1.4 \times 10^{10}$ & 62.5 & 35 & $3.6 \times 10^{6}$ & 1.27 & 4000 & 20000 & 100 & 0.529 & $\mathrm{~B}$ \\
\hline m10xe & $1.1 \times 10^{10}$ & 58.6 & 38 & $1.3 \times 10^{7}$ & 1.90 & 4000 & 20000 & 100 & 0.488 & B \\
\hline $\operatorname{m} 10 x e_{C}$ & $1.0 \times 10^{10}$ & 57.0 & 34 & $1.8 \times 10^{7}$ & 3.00 & 4000 & 20000 & 100 & 0.474 & B \\
\hline $\mathrm{m} 10 x \mathrm{x}_{\mathrm{D}}$ & $8.9 \times 10^{9}$ & 53.9 & 34 & $3.6 \times 10^{6}$ & 1.47 & 4000 & 20000 & 100 & 0.482 & B \\
\hline $\mathrm{m} 10 \mathrm{xg}_{\mathrm{A}}$ & $1.5 \times 10^{10}$ & 64.4 & 40 & $1.9 \times 10^{7}$ & 2.20 & 4000 & 20000 & 100 & 0.465 & $\mathrm{~B}$ \\
\hline \multicolumn{11}{|c|}{ Bright Dwarfs (20) } \\
\hline m10xa & $1.9 \times 10^{10}$ & 69.4 & 45 & $7.6 \times 10^{7}$ & 3.18 & 4000 & 20000 & 100 & 0.453 & B \\
\hline m10xb & $2.2 \times 10^{10}$ & 73.5 & 42 & $3.3 \times 10^{7}$ & 2.39 & 4000 & 20000 & 100 & 0.480 & $\mathrm{~B}$ \\
\hline m10xc & $3.2 \times 10^{10}$ & 82.9 & 48 & $1.2 \times 10^{8}$ & 3.26 & 4000 & 20000 & 100 & 0.451 & B \\
\hline m10xd & $3.9 \times 10^{10}$ & 88.5 & 53 & $6.8 \times 10^{7}$ & 4.04 & 4000 & 20000 & 100 & 0.437 & $\mathrm{~B}$ \\
\hline m10xe & $4.5 \times 10^{10}$ & 93.6 & 56 & $3.3 \times 10^{8}$ & 4.17 & 4000 & 20000 & 100 & 0.448 & $\mathrm{~B}$ \\
\hline m10xf & $5.2 \times 10^{10}$ & 97.7 & 58 & $1.3 \times 10^{8}$ & 3.33 & 4000 & 20000 & 100 & 0.453 & $\mathrm{~B}$ \\
\hline m10xg & $6.2 \times 10^{10}$ & 103 & 65 & $4.6 \times 10^{8}$ & 3.98 & 4000 & 20000 & 100 & 0.443 & B \\
\hline $\mathrm{m} 10 \mathrm{xh}$ & $7.4 \times 10^{10}$ & 110 & 68 & $5.4 \times 10^{8}$ & 6.04 & 4000 & 20000 & 100 & 0.434 & B \\
\hline $\mathrm{m} 10 \mathrm{xh}_{\mathrm{A}}$ & $1.5 \times 10^{10}$ & 63.9 & 38 & $5.0 \times 10^{7}$ & 3.14 & 4000 & 20000 & 100 & 0.464 & B \\
\hline m10xi & $7.6 \times 10^{10}$ & 111 & 64 & $4.5 \times 10^{8}$ & 5.16 & 4000 & 20000 & 100 & 0.441 & B \\
\hline $\mathrm{m} 10 \mathrm{z}$ & $3.5 \times 10^{10}$ & 90.5 & 49 & $4.9 \times 10^{7}$ & 3.20 & 2100 & 10000 & 43 & 0.370 & $\mathrm{D}$ \\
\hline $\mathrm{m} 11 \mathrm{a}$ & $4.0 \times 10^{10}$ & 95.0 & 52 & $1.2 \times 10^{8}$ & 2.63 & 2100 & 10000 & 43 & 0.314 & $\mathrm{D}$ \\
\hline $\mathrm{m} 11 \mathrm{~b}$ & $4.1 \times 10^{10}$ & 95.6 & 59 & $1.1 \times 10^{8}$ & 2.39 & 2100 & 10000 & 43 & 0.314 & $\mathrm{D}$ \\
\hline $\mathrm{m} 11 \mathrm{c}$ & $1.4 \times 10^{11}$ & 145 & 80 & $8.5 \times 10^{8}$ & 2.78 & 2100 & 10000 & 43 & 0.673 & $\mathrm{~F}$ \\
\hline $\mathrm{m} 11 \mathrm{~d}$ & $2.7 \times 10^{11}$ & 179 & 88 & $3.8 \times 10^{9}$ & 6.01 & 7100 & 35000 & 40 & 0.502 & $\mathrm{E}$ \\
\hline $\mathrm{m} 11 \mathrm{e}$ & $1.4 \times 10^{11}$ & 146 & 83 & $1.4 \times 10^{9}$ & 3.36 & 7100 & 35000 & 40 & 0.481 & $\mathrm{E}$ \\
\hline $\mathrm{m} 11 \mathrm{~h}$ & $1.8 \times 10^{11}$ & 157 & 90 & $3.8 \times 10^{9}$ & 3.92 & 7100 & 35000 & 40 & 0.503 & $\mathrm{E}$ \\
\hline $\mathrm{m} 11 \mathrm{i}$ & $7.0 \times 10^{10}$ & 114 & 62 & $8.9 \times 10^{8}$ & 3.35 & 7100 & 35000 & 40 & 0.548 & $\mathrm{E}$ \\
\hline $\mathrm{m} 11 \mathrm{q}$ & $1.6 \times 10^{11}$ & 153 & 80 & $6.3 \times 10^{8}$ & 2.35 & 7100 & 35000 & 40 & 0.523 & $\mathrm{D}$ \\
\hline $\mathrm{m} 11 \mathrm{q}_{880}$ & $1.5 \times 10^{11}$ & 114 & 80 & $3.7 \times 10^{8}$ & 2.83 & 880 & 4400 & 20 & 0.225 & $\mathrm{E}$ \\
\hline \multicolumn{11}{|c|}{ Milky Way-Mass (12) } \\
\hline $\mathrm{m} 12 \mathrm{~b}$ & $1.1 \times 10^{12}$ & 224 & 183 & $9.4 \times 10^{10}$ & 2.66 & 7100 & 35000 & 40 & 0.437 & G \\
\hline $\mathrm{m} 12 \mathrm{c}$ & $1.1 \times 10^{12}$ & 219 & 157 & $6.5 \times 10^{10}$ & 3.37 & 7100 & 35000 & 40 & 0.461 & G \\
\hline $\mathrm{m} 12 \mathrm{f}$ & $1.3 \times 10^{12}$ & 237 & 184 & $8.9 \times 10^{10}$ & 3.60 & 7100 & 35000 & 40 & 0.471 & $\mathrm{~F}$ \\
\hline $\mathrm{m} 12 \mathrm{i}$ & $9.4 \times 10^{11}$ & 210 & 162 & $7.0 \times 10^{10}$ & 2.80 & 7100 & 35000 & 40 & 0.496 & I \\
\hline $\mathrm{m} 12 \mathrm{~m}$ & $1.2 \times 10^{12}$ & 227 & 187 & $1.3 \times 10^{11}$ & 4.88 & 7100 & 35000 & 40 & 0.439 & $\mathrm{~F}$ \\
\hline $\mathrm{m} 12 \mathrm{r}$ & $9.0 \times 10^{11}$ & 211 & 136 & $1.9 \times 10^{10}$ & 4.37 & 7100 & 35000 & 40 & 0.476 & $\mathrm{H}$ \\
\hline $\mathrm{m} 12 \mathrm{w}$ & $9.5 \times 10^{11}$ & 215 & 157 & $5.5 \times 10^{10}$ & 3.04 & 7100 & 35000 & 40 & 0.507 & $\mathrm{H}$ \\
\hline $\mathrm{m} 12 \mathrm{z}$ & $7.3 \times 10^{11}$ & 195 & 130 & $2.2 \times 10^{10}$ & 4.71 & 4200 & 22000 & 33 & 0.383 & $\mathrm{G}$ \\
\hline Thelma & $1.1 \times 10^{12}$ & 220 & 178 & $7.7 \times 10^{10}$ & 4.36 & 4000 & 20000 & 32 & 0.366 & G \\
\hline Louise & $8.5 \times 10^{11}$ & 203 & 159 & $2.7 \times 10^{10}$ & 3.27 & 4000 & 20000 & 32 & 0.359 & $\mathrm{G}$ \\
\hline Romeo & $1.0 \times 10^{12}$ & 222 & 188 & $7.3 \times 10^{10}$ & 4.18 & 3500 & 20000 & 31 & 0.329 & G \\
\hline Juliet & $8.7 \times 10^{11}$ & 209 & 164 & $3.7 \times 10^{10}$ & 2.14 & 3500 & 20000 & 31 & 0.339 & G \\
\hline
\end{tabular}


Table E2. Resulting profile fits.

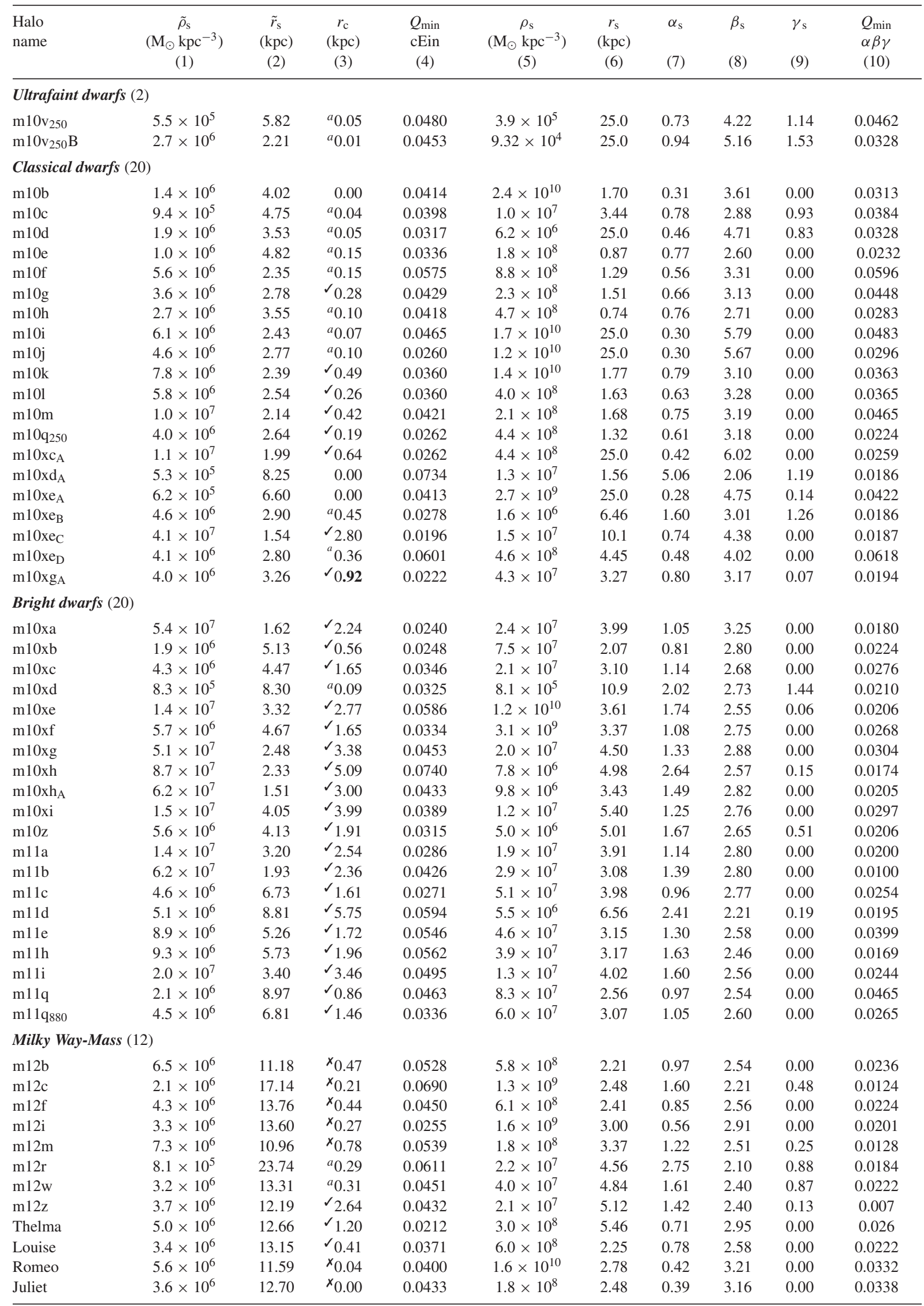


(3) $r_{\mathrm{c}}$ : The physical core radius of the dark matter profile fitted as a free parameter for the core-Einasto profile, equation (8).

(4) $Q_{\text {min, cEin }}$ The quoted goodness of fit parameter for the coreEinasto fit, i.e. equation (11).

(5) $\rho_{\mathrm{s}}$ : The scale density fitted as a free parameter for the $\alpha \beta \gamma$ profile.

(6) $(\alpha, \beta, \gamma)$ : The three characteristic slopes fitted as a free parameter for the $\alpha \beta \gamma$-profile.

(7) $Q_{\min , \alpha \beta \gamma}$ : The quoted goodness of fit parameter for the $\alpha \beta \gamma$ profile fit, i.e. equation (11).
For the quoted core radii (column 3 in Table E2), the symbols are defined as follows: $(\checkmark)$ - Verified location of dark matter core in the simulated profile; $(\boldsymbol{X})$ - Improper value of dark matter core in the simulated profile if one is physically present; $(a)$ - Dark matter core radius fitted inside the region of conservative numerical convergence, i.e. $r_{\mathrm{c}}<r_{\text {conv }}$. The exact meaning of these results are discussed in more properly in Section 4.4.

This paper has been typeset from a $\mathrm{T}_{\mathrm{E}} \mathrm{X} / \mathrm{L} \mathrm{T} \mathrm{E} \mathrm{X}$ file prepared by the author. 